\title{
Single-cell atlas of a non-human primate reveals new pathogenic mechanisms of COVID-19
}

Lei Han ${ }^{1,2,15}$, Xiaoyu Wei $e^{1,2,3,15}$, Chuanyu Liu"1,2,14,15, Giacomo Volpe ${ }^{4,15}$, Zhifeng Wang ${ }^{1,2,15}$, Taotao Pan ${ }^{1,2,3}$, Yue Yuan ${ }^{1,2,3}$, Ying Lei ${ }^{1,2,14}$, Yiwei Lai $^{4}$, Carl Ward ${ }^{4}$, Yeya $\mathrm{Yu}^{1,2,5}$, Mingyue Wang ${ }^{1,2,3}$, Quan $\mathrm{Shi}^{1,2,6}$, Tao $\mathrm{Wu}^{1,2}$, Liang $\mathrm{Wu}^{1,2,3}$, Ya $\mathrm{Liu}^{1,2}$, Chunqing Wang ${ }^{1,2,3}$, Yuanhang Zhang $^{1,2,3}$, Haixi Sun ${ }^{1,2}$, Hao $\mathrm{Yu}^{1,2}$, Zhenkun Zhuang ${ }^{1,2,7}$, Tingting Tang ${ }^{1,2}$, Yunting Huang ${ }^{2}$, Haorong $\mathrm{Lu}^{2}$, Liqin $\mathrm{Xu}^{1,2,6}$, Jiangshan $\mathrm{Xu}^{1,2,3}$, Mengnan Cheng ${ }^{1,2,3}$, Yang $\mathrm{Liu}^{1,2,3}$, Chi Wai Wong ${ }^{8}$, Tao Tan ${ }^{9}$, Weizhi $\mathrm{Ji}^{9}$, Patrick H. Maxwell ${ }^{12}$, Huanming Yang ${ }^{1,10}$, Jian Wang ${ }^{1,10}$, Shida Zhu $^{1,2}$,

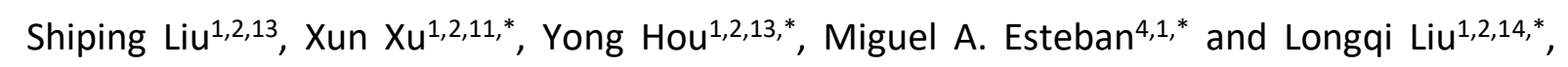
South China Greater Bay Area-Single Cell Consortium (SC-GBA-C)

${ }^{1}$ BGI-Shenzhen, Shenzhen 518083, China

${ }^{2}$ China National GeneBank, BGI-Shenzhen, Shenzhen 518120, China

${ }^{3}$ BGI Education Center, University of Chinese Academy of Sciences (CAS), Shenzhen 518083, China

${ }^{4}$ Laboratory of Integrative biology, Guangzhou Institutes of Biomedicine and Health, CAS, Guangzhou 510530, China

${ }^{5}$ BGI College, Zhengzhou University, Zhengzhou 450000, China

${ }^{6}$ Laboratory of Genomics and Molecular Biomedicine, Department of Biology, University of Copenhagen, Copenhagen 2200, Denmark

${ }^{7}$ School of Biology and Biological Engineering, South China University of Technology, Guangzhou 510006, China

${ }^{8}$ Huazhen Biosciences, Guangzhou 510900, China

${ }^{9}$ Yunnan Key Laboratory of Primate Biomedical Research, Institute of Primate Translational Medicine, Kunming University of Science and Technology, Kunming 650500, China

${ }^{10}$ James D. Watson Institute of Genome Science, Hangzhou, 310008, China

${ }^{11}$ Institute for Stem Cells and Regeneration, CAS, Beijing 100101, China

${ }^{12}$ Cambridge Institute for Medical Research, Department of Medicine, University of Cambridge, Cambridge CB2 OXY, United Kingdom

${ }^{13}$ Shenzhen Key Laboratory of Single-Cell Omics, BGI-Shenzhen, Shenzhen 518120, China 
${ }^{14}$ Shenzhen Bay Laboratory, Shenzhen 51800, China

33

${ }^{15}$ These authors contributed equally

34

35

*Correspondence:

xuxun@genomics.cn

(X.X.),

houyong@genomics.cn

(Y.H.),

miguel@gibh.ac.cn (M.A.E.), liulongqi@genomics.cn (L.L.)

37

Key words:

39 Single-cell transcriptomics, non-human primate, COVID-19, SARS-CoV-2, ACE2, TMPRSS2,

40 kidney, Interleukin 6, STAT transcription factors, immune cell exhaustion

\section{Bullet points:}

43 1-We generated a single-cell transcriptome atlas of 9 monkey tissues to study COVID-19.

442 -ACE2 ${ }^{+}$TMPRSS2 ${ }^{+}$epithelial cells of lung, kidney and liver are targets for SARS-CoV-2.

$453-A C E 2$ correlation analysis shows IDO2 and ANPEP as potential therapeutic opportunities.

46 4-We unveil a link between IL6, STAT transcription factors and boosted SARS-CoV-2 entry.

47

48 


\section{ABSTRACT}

50

51 Stopping COVID-19 is a priority worldwide. Understanding which cell types are targeted by

52 SARS-CoV-2 virus, whether interspecies differences exist, and how variations in cell state

53 influence viral entry is fundamental for accelerating therapeutic and preventative

54 approaches. In this endeavor, we profiled the transcriptome of nine tissues from a Macaca

55 fascicularis monkey at single-cell resolution. The distribution of SARS-CoV-2 facilitators, ACE2

56 and TMRPSS2, in different cell subtypes showed substantial heterogeneity across lung,

57 kidney, and liver. Through co-expression analysis, we identified immunomodulatory proteins

58 such as IDO2 and ANPEP as potential SARS-CoV-2 targets responsible for immune cell

59 exhaustion. Furthermore, single-cell chromatin accessibility analysis of the kidney unveiled a

60 plausible link between IL6-mediated innate immune responses aiming to protect tissue and

61 enhanced ACE2 expression that could promote viral entry. Our work constitutes a unique

62 resource for understanding the physiology and pathophysiology of two phylogenetically close

63 species, which might guide in the development of therapeutic approaches in humans. 


\section{INTRODUCTION}

As the distance between humans and wild animal habitats diminishes due to uncontrolled human expansion, a series of zoonotic diseases with high mortality rates have emerged. For instance, the recent outbreak of Ebola in Africa, which killed over 5,000 people, was most likely spread from bats and primates to humans ${ }^{1}$. The current outbreak of coronavirus disease 2019 (COVID-19) caused by the coronavirus severe acute respiratory syndrome coronavirus 2 $(\mathrm{SARS}-\mathrm{CoV}-2)^{2}$ is not the only example of coronaviruses that have recently passed from animals to humans. Coronaviruses are a family of RNA viruses that typically cause respiratory tract infections in humans, yet they are frequently in the reservoir of wild animals with no disease ${ }^{3}$. For example, the common cold is often (10-15\%) caused by a coronavirus (e.g. HCoV229E and HCoV-OC43) ${ }^{4}$. However, coronaviruses can also lead to severe and life-threatening diseases. In the early 2000s a coronavirus called SARS-CoV, believed to be passed from bats to humans in South East Asia, caused more than 700 deaths from around 8,000 confirmed cases worldwide ${ }^{5}$. Since 2012, another zoonotic coronavirus believed to have passed from camels to humans in the Middle East was designated as Middle East Respiratory Syndrome (MERS) ${ }^{6}$. To date, there have been over 2,500 confirmed cases of MERS with over 800 deaths. While SARS appears to have been eradicated, MERS cases are sporadic and human to human spread is limited ${ }^{4}$.

As of $21^{\text {st }}$ April 2020, COVID-19² has become a global pandemic with more than $\sim 2,500,000$ confirmed cases and over 170,000 deaths. Due to its high infectivity rate and the high level of intensive care that many patients need, COVID-19 has overwhelmed national health services and destabilized the world. One important reason is that many people who are positive for the virus show mild symptoms ${ }^{7,8}$, leading to unnoticed spread of the virus. The current worldwide emergency, possibility of continued expansion to less developed countries, risk of virus mutations and the perpetuation beyond this season has made it imperative to stop the trajectory of virus spreading. Developing drugs and preventative vaccines are ongoing but to warrant success it is necessary to have more knowledge about the disease mechanisms. So far, little is known except for the viral binding via angiotensin converting enzyme 2 (ACE2) and subsequent priming by type 2 transmembrane serine protease 2 (TMPRSS2) protease, which are shared mechanisms with SARS and MERS ${ }^{9,10}$. To test experimental treatments, animal models close to humans are necessary due to sequence 
variation of ACE2 and changes in the proportions of cell subtypes in organs between species. For these reasons, it is essential to have a species close to human to study COVID-19. In this regard, monkey experiments have shown that infection with SARS-CoV-2 produces clinical manifestations similar to COVID-19 patients ${ }^{11}$. Another study demonstrated that infection with SARS-CoV-2 in monkeys is preventable by acquired immunity, answering one of the outstanding questions about the disease $\mathrm{e}^{12}$.

Issues about the proportions of cell types within organs between species and their crosstalk can be addressed effectively through single-cell profiling technologies, in particular single-cell RNA-sequencing (RNA-seq) and single-cell assay for transposase accessible chromatin-sequencing (ATAC-seq). Yet, although human data are accumulating ${ }^{13}$, monkey data are still scarce. The comparison between human and monkey data will be crucial for advancing our knowledge of COVID-19. Here, we provide a high-resolution single-cell atlas of nine organs/tissues (lung, kidney, pancreas, brain, parotid, liver, thyroid, aorta artery, and blood) in monkey, encompassing 215,334 cells. By comparing the expression of SARS related targets in monkey and human, we have identified cell-to-cell similarities as expected. Crucially, we also discovered stark differences in ACE2 expression between these two species, for example in the ciliated vs pulmonary alveolar type 2 cells of the lung and hepatocytes in liver. We also observed that ACE2 is heterogeneous among different epithelial cell subtypes across these organs/tissues, suggesting that variations in cell state could influence viral entry. Supporting this, single-cell ATAC-seq of monkey kidney identified regulatory elements driven by signal transducer and activator of transcription factor 1 and 3 (STAT1 and 3) and interferon regulatory factor 1 (IRF) in the ACE2 locus. This suggests that cytokines, particularly interleukin 6 (IL6), aiming to induce a tissue protective response can exacerbate the disease by aiding viral entry into target cells. Additionally, through correlation analysis with ACE2 expression, we have identified several potential candidates involved in COVID-19 pathophysiology, such as Transmembrane protein 27 (TMEM27), Indoleamine 2,3dioxygenase 2 (IDO2), DnaJ heat shock protein family (Hsp40) member C12 (DNAJC12) and Alanyl aminopeptidase N (ANPEP). These targets may offer therapeutic opportunities.

Taken together, our data constitute a unique resource which could aid the scientific community in the fight against SARS-CoV-2. From a wider perspective, this will also be instrumental for systematic comparative studies aimed at understanding physiological and pathophysiological differences between monkey and other species, in particular, human. 
Cellular heterogeneity of nine non-human primate tissues assessed by single-cell RNA-seq.

We profiled, at the single-cell level, the transcriptome of the model organism cynomolgus monkey (Macaca fascicularis), as it is phylogenetically close to human and this could help advance our knowledge of human physiology and disease. As proof of principle, we decided to use our data to understand what cell types are mainly targeted by SARS-CoV-2 and how this could trigger the clinical features that have been lethal in a number of patients. For this study, we used a six-year-old female monkey in which we profiled nine different organs (Fig. 1a). These included lung, liver and kidney as the known affected organs by the closely related SARS-CoV infection ${ }^{14}$, and have been reported to have high ACE2 expression in human ${ }^{15}$. Peripheral blood mononuclear cells (PBMC) were added because altered immune responses are thought to be detrimental in the disease ${ }^{16}$. Neocortex was chosen because of the clinical symptoms which involve loss of smell and taste suggesting that the central nervous system may be targeted ${ }^{17}$. The parotid gland was chosen on the basis that saliva is one of the main means of infection spread. Additionally, we selected aorta, thyroid and pancreas.

We employed a high-throughput platform recently developed in-house, DNBelab C4, which is a scalable and cost-effective approach for microfluidic droplet-based approach ${ }^{18}$. Except for PBMC sequencing, which was performed using cells in suspension, the sequencing for all the other organs was done using single-nucleus library preparations. Following euthanasia, the selected organs were extracted, single-nucleus/cell suspensions were obtained and then used for library preparation. A total of 40,226 liver, 45,286 kidney, 36,421 pancreas, 44,355 parotid gland, 12,822 lung, 7,877 thyroid, 6,361 neocortex, 2,260 aorta nuclei and 19,726 PBMCs passed quality control and were used for downstream analysis

In a global view of our single-cell dataset, each organ clustered separately, with the exception of a few cell types such as macrophages, adipocytes and endothelial cells, which were shared between different organs (Fig. 1b). We performed Uniform Manifold Approximation and Projection (UMAP) on the 215,334 cells and identified 44 major clusters 
cluster 13, pancreatic acinar cells in cluster 26 and parotid acinar cells in cluster 30 (Fig. 1c,

Extended Data Fig. 1c). We next performed clustering and differential gene expression analysis to dissect the cellular composition of each individual organ. These analyses confirmed the typical patterns of cell heterogeneity for all the organs/tissues. When examining the lung tissue, we defined 10 major clusters with specific molecular markers, including ciliated cells, macrophages, cycling macrophages, smooth muscle cells, fibroblasts, pericytes, pulmonary alveolar (pneumocytes) type 1 and type 2, endothelial and club cells (Extended Data Fig. 2a). The kidney consisted of 11 clusters, those being podocytes, thick ascending limb cells, proximal tubule cells, intercalated cells 1 and 2, connecting tubule cells, distal convoluted tubule cells, stomal cells, thin limb cells, principal cells and endothelial cells (Extended Data

Fig. 2b). Analysis of liver tissue revealed hepatocytes to be the larger cell population, while other clusters consisted of cholangiocytes, macrophages (Kupffer cells), natural killer-T (NKT) cells, endothelial cells and hepatic stellate cells (Extended Data Fig. 2c). Inspection of PBMC clustering revealed large populations of $\mathrm{B}$ cells, $\mathrm{CD} 4^{+}, \mathrm{CD} 8^{+}$naïve and $\mathrm{CD} 8^{+}$memory $\mathrm{T}$ cells, together with smaller populations of natural killer (NK) cells, dendritic cells, CD16 $6^{+}$and $\mathrm{CD} 14^{+}$ monocytes (Extended Data Fig. 2d). Likewise, the neocortex contained excitatory neurons, astrocytes, microglia, parvalbumin (PVALB), somatostatin-expressing neurons (SST), synaptic vesicle glycoprotein-expressing cells (SV2C), vasoactive intestinal polypeptide-expressing neurons (VIP), oligodendrocytes and oligodendrocyte precursor cells (Extended Data Fig. 2e). Parotid gland instead was composed of a large cluster of serous acinar cells together with small clusters of macrophages, stromal cells, myoepithelial cells, striated duct cells, mucous acinar cells and intercalated duct cells (Extended Data Fig. 2f). Aorta cells could be further divided into adipocytes, endothelial cells, myofibroblasts and a large proportion of smooth muscle cells (Extended Data Fig. 2g). Our clustering also demonstrated that most of the thyroid gland is composed of follicular cells, with smaller populations of adipocytes, endothelial cells, stromal and smooth muscle cells (Extended Data Fig. 2h). Finally, our data showed the largest population of the pancreas to be acinar cells, while smaller clusters were comprised of stromal, ductal, and islet cells (alpha and beta), together with a population that could not be assigned to any known cell type (Extended Data Fig. 2i). 


\section{ACE2 and TMPRSS2 single-cell expression landscape in a non-human primate.}

Recent studies have reported that, similarly to SARS-CoV, the capacity of SARS-CoV-2 virus to infect host cells relies on viral spike (S) protein binding to ACE2 entry receptor ${ }^{9}{ }^{10}$, which is involved in the control of blood pressure through the renin-angiotensin system ${ }^{19}$. This phenomenon is primed by the multifunction serine protease TMPRSS2 ${ }^{20}$. Accordingly, double positive $\left(\mathrm{ACE2}^{+} / \mathrm{TMPRSS2}^{+}\right.$) cells have higher risk of infection by SARS-CoV-2. Although immunohistological studies have demonstrated localization of these two proteins in the respiratory tract ${ }^{21}$, it is unclear which cell subtypes express these genes and how homogenous the expression among a specific cell subtype is. Also, comprehensive information about other cell types and organs that express these two proteins and could be targeted by the virus in human or monkey is lacking.

We inspected our data to see how widespread and homogenous ACE2 expression was in the monkey tissues. As expected, ACE2 was detected in several lung clusters, mainly ciliated cells, club cells and pulmonary alveolar type 2 cells (Fig. 2a, 2d, 3a upper panel), whereas in the kidney, ACE2 was primarily present in proximal tubule cells (Fig. 2a, 2d, 3b upper panel). The latter is consistent with reports describing that a significant number of COVID-19 patients display altered kidney function ${ }^{15,22}$. Interestingly, ACE2 expression was heterogenous among these cell subtypes in both lung and kidney. In the liver, ACE2 was mostly expressed in cholangiocytes, with a smaller degree of expression also found in hepatocytes (Fig. 2a, 2d, 3c upper panel). Notably, the closely related SARS-CoV caused liver injury due to hepatitis in some patients ${ }^{23}$, suggesting that the liver may also be a direct target for SARS-CoV-2. A small proportion of $\mathrm{ACE2}^{+}$was also observed in pancreatic islet cells (Fig. 2a, 2d, Extended Data Fig. 3a). In contrast, little or no expression was observed in thyroid, neocortex, parotid and PBMC (Fig. 2a, 2d, Extended Data Fig. 3a). Negligible ACE2 expression in the neocortex suggests that other tissues may be affected by SARS-CoV-2 that cause loss of taste and smell, regarding the latter in particular the olfactory epithelium.

TMPRSS2 displayed more broadly expressed across cell types in multiple tissues, although it was highest in kidney cells. However, in contrast to ACE2, its expression was highest in the distal convoluted tubule, thin limb, intercalated and principal cell 1 and 2 kidney clusters (Fig. 2b, 2d, 3b lower panel, Extended Data Fig. 3b). Additionally, significant TMPRSS2 was observed in both parotid and pancreatic acinar cells, thyroid follicular cells, cholangiocytes and in several lung clusters (Fig. 2b, 2d, Extended Data Fig. 3). We then 
determined which cells co-expressed both genes $\left(A C E 2^{+} / T_{M P R S S}{ }^{+}\right)$. Notably, the largest overlap between ACE2 and TMPRSS2 was observed in the ciliated and club cell clusters of the lung and to a lesser extent the proximal tubule cells of the kidney (Fig. 2c, 2e). A smaller overlap was also observed in cholangiocytes and in pancreatic islet cells (Fig. 2c, 2e).

Therefore, our data show that ACE2 and TMPRSS2 are expressed in a variety of cell types, mainly epithelial cells, within the nine monkey organs/tissues (Supplementary Table 2a). The observed heterogeneity of $A C E 2$ in these cell subtypes also suggests that variations in cell state (e.g. differentiation state, stimulation state or topographical distribution) cause heterogenous expression across an individual tissue. These observations may provide important clues about COVID-19 pathogenesis and symptomatology.

\section{Comparative analysis of ACE2 and TMPRSS2 expression in human and non-human primate.}

Given the heterogeneous nature of $A C E 2$ and TMPRSS2 expression within monkey tissues, we decided to investigate similarities and differences between human and monkey. For this purpose, we retrieved publicly available data from single-cell studies in human (see methods). TMPRSS2 distribution was similar in cell subtypes of lung, kidney and liver between human and monkey (Fig. 3d-3f). However, strikingly, ACE2 showed distinct patterns among cell subtypes in all three organs between the two species (Fig. 3d-3f). The biggest differences were observed in ciliated cells of the lung, which had the highest expression of ACE2 in monkey, and pulmonary alveolar type 2 cells, which had the highest expression of ACE2 in human. The function of ciliated cells is to move substances (e.g. cell debris and toxic material) across the surface of the respiratory tract and are commonly targeted by respiratory viruses, whereas pulmonary alveolar type 2 cells have regenerative properties, are crucial for alveolar homeostasis and produce surfactant ${ }^{24,25}$. In the kidney of both monkey and human, the highest ACE2 expression was in proximal tubule cells (Fig. 3e), which are responsible for electrolyte and nutrient reabsorption. However, renal endothelial cells had higher expression in monkey compared to human. In liver, cholangiocytes had similarly high ACE2 expression in monkey and human, but hepatocytes showed higher expression and more positive cells in the human (Fig. 3f). Considering the heterogenous expression of ACE2 within the proximal tubule cells in monkey, we revisited the previously analyzed data and were able to sub cluster this population of cells into two (S1 and S3) based on the expression of SLC5A2 and SLC7A13 ${ }^{26}$

(Extended Data Fig. 4, Supplementary Table 2b). These two genes are sodium and glucose 
cotransporters involved in glucose reabsorption in the kidney ${ }^{27,28}$. We did not include thyroid, pancreas or aorta in these analyses because of lack of high-quality available human single-cell datasets. As for the neocortex and PBMC, they have little to no expression of ACE2 in human (data not shown).

These differences in ACE2 expression across cell subtypes in the lung, kidney and liver in monkey and human raise the possibility that infection with SARS-CoV-2 in the two species will have different effects.

ACE2 correlation analysis across cell types reveals potential therapeutic targets.

To shed light on potential mechanisms that could facilitate ACE2-mediated SARS-CoV-2 infection, we performed an analysis of the Pearson's correlation coefficient, based on gene expression in the 44 cell subtypes, to determine what genes are co-regulated with ACE2 in monkey tissues. Correlated genes were considered as those displaying a coefficient higher than 0.6 with an adjusted $P$ value $<0.001$. Using these criteria, we observed several genes with marked correlation, including genes that belong to metabolic and developmental pathways and genes involved in the cellular response to xenobiotic stimuli (Fig. $\mathbf{4 a}, \mathbf{b}$ ). The highest correlation was observed for transmembrane protein $27(T M E M 27$, cor $=0.84)$, a protein involved in trafficking amino acid transporters to the apical brush border of kidney proximal tubules ${ }^{29}$. This is unsurprising considering that TMEM27 is an important paralog of ACE2, and high expression was restricted to kidney cells. DnaJ heat shock protein family (Hsp40) member C12 (DNAJC12, cor $=0.78)$, a gene with a role in immune response processes $^{30}$, had a distribution like TMEM27. Importantly, we also observed high correlation with Indoleamine 2,3-dioxygenase $2(I D O 2$, cor $=0.77)$, a gene with abundant expression in kidney and liver cells that was also expressed in the lung and other organs. IDO2 functions during the early phases of immune responses and promotes inflammatory autoimmunity 31 , 32. ANPEP, which encodes for alanyl aminopeptidase $\mathrm{N}$, was also co-expressed with ACE2 in kidney, liver and to a lesser extent in lung too (cor = 0.64), like IDO2 (Fig. 4c, d). Interestingly, ANPEP has also been shown to be participate in immune responses, virus receptor activity and in mediating virus entry into host cells ${ }^{33,34}$.

These data highlight potential therapeutic targets to help in the fight against SARSthat they modulate and/or are directly involved in viral entry. Alternatively, depletion of cells 
expressing IDO2 and ANPEP through a cytopathic effect of the virus could trigger an uncontrolled immune response and contribute to the immune cell exhaustion observed in COVID-1935.

\section{Epigenetic regulation of ACE2 in each cell subtype of monkey kidney.}

To understand whether epigenetic mechanisms underlie the heterogeneity of ACE2 expression in the kidney, as representative for other organs, we employed DNBelab C4 technology to perform high-throughput single-cell ATAC-seq (Fig. 5a). After filtering, 6,353 nuclei were used for downstream analysis (Extended Data Fig. 5a, b, Supplementary Table 4). We integrated these data with the kidney transcriptomic data described in Fig. 1 and proceeded to perform Louvain clustering to map all the different cell types within the dataset (Fig. 5b). Consistent with the transcriptomic data, our epigenomic mapping identified thick ascending limb cells and proximal tubule cells as the largest kidney clusters (Extended Data

Fig. 2b). Similarly, smaller clusters of podocytes, principal, intercalated, connected tubule, distal convoluted tubule, thin limb, endothelial and stromal cells were detected (Fig. 5c,

Extended Data Fig. 5c). Analysis of open chromatin regions revealed discrete peaks in the ACE2 locus, with the highest signal detected in proximal tubule cells $\mathrm{S} 1$ and $\mathrm{S} 3$, which are also the highest ACE2-expressing cells (Fig. 5d). Our approach failed to detect significant signal enrichment in the ACE2 locus in endothelial cells, possibly related to the low level of expression (Fig. $\mathbf{5 d}$ ). Within the cells of the kidney we observed the highest percentage of $A C E 2^{+}$cells in the proximal tubule $\mathrm{S} 3$, with a lower percentage in the proximal tubule $\mathrm{S} 1$ and endothelial cells (Fig. 5e). Motif analysis within the open chromatin regions in $\mathrm{ACE2}^{+}$cells demonstrated that these regions were preferentially enriched in STAT1 and 3 and IRF1 binding sites (Fig. 5f). These findings suggested that tissue protective cytokines including IL5, IL6, EGF and interferons are acting on these proximal tubule cells S3 to induce ACE2. We focused on IL6 because a recent clinical trial has been started that uses anti-IL6 receptor (IL6R) antibodies in the treatment of COVID-19 (http://www.chictr.org.cn/showprojen.aspx?proj=49409). IL6 is a potent regulator of immune responses and can be produced by a variety of interstitial cells including fibroblasts, endothelial cells and more importantly tissue macrophages ${ }^{36}$. Interestingly, we also noticed 

expression that can exacerbate COVID-19 disease progression due to increased viral entry and dissemination.

\section{DISCUSSION}

Mammalian tissues and organs are composed of many different cell types that can vary in abundance and cell state. Tissue heterogeneity is only beginning to be unraveled thanks to the advent of single-cell profiling technologies that allow us to precisely map transcriptomic and epigenomic programs. These technologies are revolutionizing our view of human physiology and disease. Great efforts are being made to generate the first version of both human and murine atlases ${ }^{13,37}$. The mouse is among the most commonly used model organisms in biomedical research but many developmental or pathological aspects are not paralleled in human. Understanding tissue and organ complexity in species that are phylogenetically close to humans is an unmet requirement.

In this study, we have generated a single-cell transcriptomic atlas of nine organs (liver, kidney, lung, pancreas, neocortex, aorta, parotic gland, thyroid and peripheral blood) from cynomolgus monkey. We used this dataset not only to provide fundamental information about the cellular composition of the different tissues tested but also as a platform to dissect the overall expression distribution of the SARS-CoV-2 entry receptor, ACE2, and its serine protease coactivator TMPRSS2 ${ }^{9,10}$. Interestingly, ACE2 was expressed in multiple epithelial tissues besides the lung, especially the kidney and liver. Other organs of epithelial origin such as the gut have also been implicated in the pathogenesis of the disease ${ }^{38}$. A consequence of this is that the SARS2-CoV-2 virus could infect these organs too, which would explain some of the reported clinical manifestations of COVID-19². By comparing our dataset with publicly available human single-cell RNA-seq data, we have also uncovered significant differences in cell subtypes expressing ACE2 between human and monkey. We showed different expression patterns for ACE2 in the lung, where the highest levels were detected in ciliated cells in monkey and pulmonary alveolar type 2 cells in human. Similarly, we observed marked differences in liver, in which monkey hepatocytes displayed higher ACE2 and a larger number of positive cells compared to the human. We do not know whether these differences will 
affect the pathogenesis of COVID-19 between these two species. Nevertheless, this is a relevant finding considering that monkeys are a preferred model for studying the effectiveness of drug treatments and of vaccines against the impending COVID-19 pandemic.

Through correlation analysis, we identified new potential mechanisms that could facilitate ACE2-mediated viral infection, including genes previously unreported in the context of SARS-CoV-2 that are involved in stimulating different types of immune responses. We observed high expression of IDO2 and ANPEP in kidney, liver and lung. Expression of these genes can be further induced by viral infection and they have been reported to be immune modulators and/or mediate viral entry ${ }^{31,33}$. These observations are relevant as they highlight new potential therapeutic vulnerabilities in the current emergency. In this respect, a number of inhibitors of ANPEP are currently being tested in several disease contexts and could serve to prevent the immune cell exhaustion often observed in many severe COVID-19 cases $^{39}$. Similarly, mesenchymal stem/stroma cells (MSC) have immunomodulatory functions that are partly related to IDO2 production ${ }^{31}$. It is tempting, thus, to speculate that cell therapies based on MSC delivery could ameliorate COVID-19 by normalizing immune function and preventing cytokine storms ${ }^{40}$.

Intriguingly, in our data, we see heterogenous expression of ACE2 within the individual cell subtypes in six out of the nine monkey organs that we analyzed, which is also the case in the three human organs analyzed. In this regard, we noticed two different cell populations in the kidney proximal tubule, one with higher ACE2 expression than the other. We performed single-cell ATAC-seq of this organ to understand whether this phenomenon has an epigenetic basis. Analysis of open chromatin regions within the ACE2 locus revealed the enrichment of STAT1, STAT3 and IRF1 binding sites. These transcription factors have important immune functions and are direct targets of tissue protective and innate immune responses such as Interleukin-6 signaling pathway and interferons. Analysis of IL6R distribution showed broad expression within different the $A C E 2^{+}$organs in monkey and human. This suggests a link between paracrine IL6 (e.g. secreted by stromal cells including tissue resident macrophages) and enhanced ACE2 expression across different organs. Higher and more widespread ACE2 expression could promote increased viral entry. This observation could be very relevant given recent reports describing clinical trials with Tocilizumab, a monoclonal antibody used for IL6R blockade in patients with rheumatoid arthritis ${ }^{41}$, for the treatment of COVID-19 
tissue damage $e^{42}$, and this may explain why elderly individuals and those with underlying

386 inflammatory conditions have more severe reactions to SARS-CoV-2 infection (Fig. 6).

387 Importantly, high IL6 levels have been detected in plasma from COVID-19 patients ${ }^{43}$. In this

388 context, the proposed enhanced affinity of SARS-CoV-2 for ACE2 compared to SARS-CoV may

389 underlie the differences in the clinical course between the two diseases ${ }^{44}$.

$390 \quad$ All these observations reveal new potential mechanisms for COVID-19, opening new

391 therapeutic avenues for disease management. However, caution should be exercised when

392 making decisions before additional experimental validation becomes available. Further

393 scrutiny of our datasets may provide new associations useful for understanding COVID-19,

394 and in general will be of utmost relevance for systematic comparisons aiming to understand

395 monkey and human tissue composition and disease vulnerabilities. 
METHODS

Ethics statement.

400 This study was approved by the Institutional Review Board on Ethics Committee of BGI

401 (permit no. BGI-IRB19125).

402

403

\section{Collection of monkey tissues.}

404

A 6-year old female cynomolgus monkey was purchased from Huazhen Laboratory Animal Breeding Centre (Guangzhou, China). The monkey was anesthetized with ketamine hydrochloride $(10 \mathrm{mg} / \mathrm{kg})$ and barbiturate $(40 \mathrm{mg} / \mathrm{kg})$ administration before being euthanized by exsanguination. Tissues were isolated and placed on the ice-cold board for dissection. Whole organs including lung, kidney, pancreas, liver, brain, thyroid, parotid gland, and aorta were cut into 5-10 pieces, respectively (50-200 mg/piece). Samples were then quickly frozen in liquid nitrogen and stored until nuclear extraction was performed. PBMC were isolated from heparinized venous blood using a Lymphoprep ${ }^{\mathrm{TM}}$ medium (STEMCELL Technologies, \#07851) according to standard density gradient centrifugation methods. PBMC were resuspended in 90\% FBS, 10\% DMSO (Sigma Aldrich, \#D2650) freezing media and frozen using a Nalgene ${ }^{\circledR}$ Mr. Frosty ${ }^{\circledR}$ Cryo $1^{\circ} \mathrm{C}$ Freezing Container (Thermo Fisher Scientific, \#5100-0001) in a $-80^{\circ} \mathrm{C}$ freezer for 24 hours before being transferred to liquid nitrogen for long-term storage.

\section{Single-nucleus/cell suspension preparation.}

We isolated nuclei as previously described ${ }^{45}$. Briefly, tissues were thawed, minced and added to lysis buffer. Lysates were filtered and resuspended in cell resuspension buffer. Frozen PBMC vials were rapidly thawed in a $37^{\circ} \mathrm{C}$ water bath for $\sim 2$ minutes, then quenched with 10 $\mathrm{ml} 37^{\circ} \mathrm{C}$ pre-warmed $1 \mathrm{X}$ phosphate-buffered saline (PBS, Thermo Fisher Scientific, \#10010031) supplemented with 10\% FBS. PBMCs were centrifuged at 500 R.C.F. for 5 minutes at room temperature. The supernatant was removed, and the cell pellet was resuspended in $3 \mathrm{ml} 37^{\circ} \mathrm{C}$ pre-warmed $1 X$ PBS containing $0.04 \%$ bovine serum albumin (BSA, Sangon Biotech, A600903), passed through a $40 \mu \mathrm{m}$ cell strainer (Falcon, \#352340) and then centrifuged at 500 R.C.F. for 5 minutes at room temperature. Nuclei or cells were resuspended with cell resuspension buffer at a concentration of 1,000 cells/ $\mu$ l for single-cell library preparation. 
431 The DNBelab C Series Single-Cell Library Prep Set (MGI, \#1000021082) was utilized as previously described (Liu et al. 2019). In brief, single-nucleus/cell suspensions were used for droplet generation, emulsion breakage, beads collection, reverse transcription, and cDNA amplification to generate barcoded libraries. Indexed single-cell RNA-seq libraries were constructed according to the manufacturer's protocol. The sequencing libraries were quantified by Qubit ${ }^{\mathrm{TM}}$ ssDNA Assay Kit (Thermo Fisher Scientific, \#Q10212). Single-cell ATACseq libraries were prepared using DNBelab C Series Single-Cell ATAC Library Prep Set (MGI, \#1000021878). DNA nanoballs (DNBs) were loaded into the patterned Nano arrays and sequenced on the ultra-high-throughput DIPSEQ T1 sequencer using the following read length: $30 \mathrm{bp}$ for read 1, inclusive of $10 \mathrm{bp}$ cell barcode 1, $10 \mathrm{bp}$ cell barcode 2 and $10 \mathrm{bp}$ unique molecular identifier (UMI), $100 \mathrm{bp}$ of transcript sequence for read 2, and $10 \mathrm{bp}$ for sample index.

\section{Single-cell RNA-seq data processing.}

Raw sequencing reads from DIPSEQ-T1 were filtered and demultiplexed using PISA (version

0.2) (https://github.com/shiquan/PISA). Reads were aligned to Macaca_fascicularis_5.0 genome using STAR (version $2.7 .4 a)^{46}$ and sorted by sambamba (version 0.7 .0$)^{47}$. Cell versus gene UMI count matrix was generated with PISA.

\section{Cell clustering and identification of cell types.}

451 Clustering analysis of the complete cynomolgus monkey tissue dataset was performed using 452 Scanpy (version 1.4$)^{48}$ in a Python environment. Parameters used in each function were manually curated to portray the optimal clustering of cells. In preprocessing, cells or nuclei were filtered based on the criteria of expressing a minimum of 200 genes and a gene which is expressed by a minimum of 3 cells or nuclei. Filtered data were In (counts per million $(\mathrm{CPM}) / 100+1)$ transformed. 3000 highly variable genes were selected according to their average expression and dispersion. The number of UMI and the percentage of mitochondrial gene content were regressed out and each gene was scaled by default options. Dimension 
is then used to detect subgroups of cells. Distinguishing differential genes among clusters were ranked (Benjamini-Hochberg, Wilcoxon rank-sum test). Cell types were manually and iteratively assigned based on overlap of literature, curated and statistically ranked genes. Each tissue dataset was portrayed using the Seurat package (version 3.1.1 ${ }^{49}$ in R environment by default parameters for filtering, data normalization, dimensionality reduction, clustering, and gene differential expression analysis. Finally, we annotated each cell type by extensive literature reading and searching for the specific gene expression pattern.

Gene correlation and Gene Ontology (GO) term enrichment analysis.

The correlation between ACE2 and other genes was drawn using Pearson correlation coefficient (PCC) with gene expression value merged from cells of the same cell types with the $\mathrm{R}$ package psych (version 1.9.12.31). To infer the biological function of highly correlated genes (cor $>0.6$ and adjusted $P$ value $<0.001$ ), we performed gene set enrichment analysis using Metascape (https://metascape.org/gp/index.html).

Differential gene expression analysis.

Differential expression analysis between proximal tubule S1 and proximal tubule S3 was performed using the FindMarkers function of the Seurat package (version 3.1.1).

Single-cell ATAC-seq data processing.

Raw sequencing reads from DIPSEQ-T1 were filtered and demultiplexed using PISA (version generated by custom script. To create a gene activity matrix, we extracted gene coordinates for cynomolgus monkey from NCBI, and extended them to $\pm 2 \mathrm{~kb}$ region around TSS. The gene activity score matrix was calculated by custom script.

\section{Single-cell ATAC-seq cell clustering and cell type identification.}

Cells with low fragments $(<1,000)$ and TSS proportion $(<0.1)$ were removed. Then, filtered data were imported into $\mathrm{R}$ and the dimensionality was reduced by latent semantic indexing. 
493

494

495

496

497

498

499

500

501

502

503

504

505

506

507

508

509

510

511

512

513

514

515

516

517

518

519

520

521

522

523

524

the single-cell ATAC-seq and single-cell RNA-seq data by the TransferData function of Seurat (version 3.1.1).

\section{Transcription factor motif enrichment analysis.}

To predict the motif footprint in peaks within the ACE2 promoter, we extracted genome sequences in the peak region with Seqkit (version 0.7.0) ${ }^{51}$. The sequences were imported into $\mathrm{R}$ and were matched with all Homo sapiens motifs form JASPAR2018 using matchMotifs function in motifmatchr packages version 1.8 .0 with default parameter.

\section{Human single-cell RNA-seq datasets.}

All human single-cell RNA-seq data matrix were obtained from publicly available dataset as described: (1) Kidney data from Stewart et al. was download from https://www.kidneycellatlas.org/52; (2) Lung data from Madissoon et al. was download from https://www.tissuestabilitycellatlas.org/53; (3) Liver data from Aizarani et al. was download from GEO at accession GSE124395 ${ }^{54}$.

\section{Code availability}

Computer code used for processing the single-cell RNA-seq and single-cell ATAC-seq will be available at https://github.com/brucepan10/NHP-COVID-19.

\section{Data availability}

All raw data have been deposited to CNGB Nucleotide Sequence Archive (accession code: CNP0000986; https://db.cngb.org/cnsa/project/CNP0000986/public/)

\section{ACKOWLEDGEMENTS}

We thank Xiaoyun Huang and Miaomiao Jiang of the Zhiyu Center for Systems Biology, Zhiyu Inc., Shenzhen for their support. This work was supported by National Natural Science Foundation of China (31900466, 31900582), Research and Development Program of China (2018YFA0106903), the Strategic Priority Research Program of the Chinese Academy of Sciences (XDA16030502) and CAS-JSPS Joint Research Projects (GJHZ2093), Natural Science 
525 Foundation of Guangdong Province, China (2018A030313379), the Shenzhen National Key

526 Laboratory of Single-Cell Omics (ZDSYS20190902093613831) and Shenzhen Bay Laboratory

527 (SZBL2019062801012). G.V. is supported by Chinese Academy of Sciences President's

528 International Fellowship for Foreign Experts (2020FSB0002). C.W. is supported by Chinese

529 Academy of Sciences President's International Fellowship Initiative for Postdoctoral

530 Researchers (2019PB0177) and Research Fund for International Young Scientists grant

531 (31950410553). This work is part of the South China Greater Bay Area-Single Cell Consortium

532 under the coordination of M.A.E, L.L., X.X. and Y.H.. This publication is part of the Human Cell

533 Atlas - www.humancellatlas.org/publications/.

534

535

536 AUTHOR CONTRIBUTIONS

537

538 L.L., M.A.E., Y.H. and X.X. conceived the idea. L.L., L.H., Y.L., S.L., X.W. and Y.Yuan. designed

539 the experiment. L.H., Y.L., S.L., X.W., Y.Y., M.C. and C.W.W. collected the tissue samples. C.L.,

540 Z.W., Y.Yuan, Y. Yu, M.W., T.W., Y.L., C.W., Y.Z., T.T., Y.H., H.L., L.X., J.X. and M.C. performed

541 the experiments. X.W., T.P., Q.S., L.W., Z.Z., Y.L., S.Z. and S.L. performed the data analysis.

542 L.L., L.H., X.W., C.L., G.V., T.P., C.W. and Y.L. prepared the figures. P.H.M provided critical

543 review of the manuscript. M.A.E., G.V., C.W., Y.L. and L.L. wrote the manuscript with input

544 from all authors. X.X., Y.H., L.L. and M.A.E supervised the entire study. All other authors

545 contributed to the work. All authors read and approved the manuscript for submission.

546

547

548 COMPETING INTERESTS

549

550 Employees of BGI have stock holdings in BGI. 
Fig. 1 | Construction of single-cell atlas across nine tissues of a Macaca fascicularis monkey. experimental pipeline for the single-cell sequencing.

b, UMAP visualization of all single cells from the dataset colored by tissue/organ (left) and number of cells from each tissue passing quality control (right).

c, UMAP visualization of each cell type colored according to 44 clusters in the first round of clustering. Cell type annotation is provided in the figure and is associated with a number indicative of every cluster. $n=215,334$ individual nuclei/cells.

562

Fig. 2 | ACE2 and TMPRSS2 expression across 44 cell clusters in monkey.

(b) expression in all single cells within our dataset.

c, UMAP projection of $A C E 2^{+} / T M P R S S 2^{+}$cells.

d, Bubble plots showing the level of expression of TMPRSS2 and ACE2 genes and the ratio of expressing cells in the indicated cell types. The color of each bubble represents the level of expression and the size indicates the proportion of expressing cells.

570

e, Barplot indicating the percentage of ACE2 and TMPRSS2 expressing cells within each cell cluster.

572

Fig. 3 | Comparative analysis of ACE2 and TMPRSS2 expression between monkey and a-c, UMAP projection of ACE2 (top) and TMPRSS2 (bottom) expression in single cells of monkey lung (a), kidney (b) and liver tissues (c). The red arrow in this panel indicates cholangiocytes. The color of the cells reflects the expression level as indicated in the scale bar. d-f, Bubble plots showing the ratio and expression of ACE2 and TMPRSS2 in the indicated cell types of lung (d), kidney (e) and liver (f) in monkey and human. The color of each bubble represents the level of expression and the size indicates the proportion of expressing cells. 

and other individual genes. The correlation coefficient for every gene ( $x$-axis) versus the adjusted $P$ value (using Benjamini-Hochberg correction; $y$-axis). The genes indicated in the plot are those with a correlation score $>0.6$ and an adjusted $P$-value $<0.001$.

587

b, Gene ontology analysis of genes that show high expression correlation with ACE2.

588

c, Scatter plots showing the association between ACE2 and the indicated genes. The correlation coefficients (Pearson $r^{2}$ ) and adjusted $P$ values are shown in the plots.

590

d, UMAP projection of expression of the indicated genes in all single cells.

591

592

Fig. 5 | Chromatin accessibility analysis reveals epigenetic regulation of ACE2 in kidney.

593 a, Schematic of experimental design for single-cell ATAC-seq of monkey.

594 b, Joint UMAP visualization of kidney single-cell ATAC (scATAC)-seq data with single-cell RNA 595 (scATAC)-seq data. c, UMAP visualization of single-cell ATAC-seq data.

596 d, IGV visualization of aggregate single-cell ATAC-seq signal in each cell type.

597 e, Ratio of $\mathrm{ACE2}^{+}$cells in each cell type of kidney.

598 f, The transcription factor motifs predicted based on DNA sequence within those regions of 599 the ACE2 locus.

600 g-h, UMAP projection of IL6R expression and cells with $I L 6 R^{+} / A C E 2^{+}$cells in all kidney single 601 cell in monkey (g) and human (h).

602

Fig. 6 | Proposed molecular mechanism for SARS-Cov-2 pathogenesis through reinforced IL6-mediated immune response in monkey and humans.

Schematic representation of potential mechanism of SARS-CoV-2 spreading through lung, kidney and liver. Kidney proximal tubule cells within the nephron have the highest expression of ACE2 receptor which facilitates virus entry. After virus contact, IL6R stimulates an immune response that, through the activation of STAT factors, potentiates the paracrine positive feedback loop that facilitates virus spreading. IL6 expression, which is higher in elderly patients and those with inflammatory conditions, is effectively targeted by anti-IL6R monoclonal antibodies leading to a more favourable disease course. 


\section{Extended Data Fig. 1 | Quality control of the single-cell RNA-seq libraries.}

618 a, Violin plot showing the number of unique molecular identifiers (UMIs) identified in each 619 tissue.

620 b, Violin plot showing the number of genes identified in each organ. (C) Heatmap showing the expression of marker genes of the indicated cell type

622

Extended Data Fig. 2 | Various cell types identified in each tissue.

a-i, UMAP visualization of cell clusters in lung (a), kidney (b), liver (c), PBMC (d), neocortex (e), parotid (f), aorta ( $(\mathbf{g})$, thyroid $(\mathbf{h})$ and pancreas (i). The name of the population corresponding to each cell cluster is indicated in every plot.

627

Extended Data Fig. 3 | ACE2 and TMPRSS2 expression in each tissue. a-b, UMAP Projection of ACE2 (a) and TMPRSS2 (b) expression in each tissue.

630 kidney.

a, UMAP visualization of single cells from the kidney tissue, colored by cell types.

b, Volcano plot showing the differentially expressed genes between proximal tubule S1 and proximal tubule S3 cells. Examples of highly variable genes are indicated.

c, UMAP projection of expression for the indicated genes in all single cells. expression level for proximal tubule S1 and proximal tubule S3 cells are indicated.

639

Extended Data Fig. 5 | Quality control of single-cell ATAC-seq data.

641 a, Number of fragments captured in all cells of the two single-cell ATAC-seq libraries.

b, Proportion of TSS fragments in all cells of the two single-cell ATAC-seq libraries. 


\section{REFERENCES}

648

1. Kock, R.A., Begovoeva, M., Ansumana, R. \& Suluku, R. Searching for the source of Ebola: the elusive factors driving its spillover into humans during the West African outbreak of 2013-2016. Rev Sci Tech 38, 113-122 (2019).

2. Zhu, N. et al. A Novel Coronavirus from Patients with Pneumonia in China, 2019. The New England journal of medicine 382, 727-733 (2020).

3. Cui, J., Li, F. \& Shi, Z.L. Origin and evolution of pathogenic coronaviruses. Nat Rev Microbiol 17, 181-192 (2019).

4. Su, S. et al. Epidemiology, Genetic Recombination, and Pathogenesis of Coronaviruses. Trends Microbiol 24, 490-502 (2016).

5. Lau, S.K. et al. Severe acute respiratory syndrome coronavirus-like virus in Chinese horseshoe bats. Proc Natl Acad Sci U S A 102, 14040-14045 (2005).

6. Reusken, C.B. et al. Middle East respiratory syndrome coronavirus neutralising serum antibodies in dromedary camels: a comparative serological study. Lancet Infect Dis 13, 859-866 (2013).

7. Chan, J.F. et al. A familial cluster of pneumonia associated with the 2019 novel coronavirus indicating person-to-person transmission: a study of a family cluster. Lancet 395, 514-523 (2020).

8. Rothe, C. et al. Transmission of 2019-nCoV Infection from an Asymptomatic Contact in Germany. The New England journal of medicine 382, 970-971 (2020).

9. Hoffmann, M. et al. SARS-CoV-2 Cell Entry Depends on ACE2 and TMPRSS2 and Is Blocked by a Clinically Proven Protease Inhibitor. Cell 181, 271-280 (2020).

10. Walls, A.C. et al. Structure, Function, and Antigenicity of the SARS-CoV-2 Spike Glycoprotein. Cell, Epub ahead of print (2020).

11. Rockx, B. et al. Comparative pathogenesis of COVID-19, MERS, and SARS in a nonhuman primate model. Science, Epub ahead of print (2020).

12. Bao, L. et al. Reinfection could not occur in SARS-CoV-2 infected rhesus macaques. bioRxiv, 2020.2003.2013.990226 (2020).

13. Han, X. et al. Construction of a human cell landscape at single-cell level. Nature (2020).

14. Hamming, l. et al. Tissue distribution of ACE2 protein, the functional receptor for SARS coronavirus. A first step in understanding SARS pathogenesis. J Pathol 203, 631-637 (2004).

15. Naicker, S. et al. The Novel Coronavirus 2019 epidemic and kidneys. Kidney Int (2020).

16. Conti, P. et al. Induction of pro-inflammatory cytokines (IL-1 and IL-6) and lung inflammation by Coronavirus-19 (COVI-19 or SARS-CoV-2): anti-inflammatory strategies. J Biol Regul Homeost Agents 34 (2020).

17. Bagheri, S.H.R. et al. Coincidence of COVID-19 epidemic and olfactory dysfunction outbreak. medRxiv, 2020.2003.2023.20041889 (2020).

18. Liu, C. et al. A portable and cost-effective microfluidic system for massively parallel single-cell transcriptome profiling. bioRxiv, 818450 (2019).

19. Turner, A.J., Hiscox, J.A. \& Hooper, N.M. ACE2: from vasopeptidase to SARS virus receptor. Trends Pharmacol Sci 25, 291-294 (2004).

20. Kim, T.S., Heinlein, C., Hackman, R.C. \& Nelson, P.S. Phenotypic analysis of mice lacking the Tmprss2-encoded protease. Mol Cell Biol 26, 965-975 (2006). 
21. Jia, H.P. et al. ACE2 receptor expression and severe acute respiratory syndrome coronavirus infection depend on differentiation of human airway epithelia. J Virol 79, 14614-14621 (2005).

22. Li, Z. et al. Caution on Kidney Dysfunctions of COVID-19 Patients. medRxiv, 2020.2002.2008.20021212 (2020).

23. Peiris, J.S. et al. Coronavirus as a possible cause of severe acute respiratory syndrome. Lancet 361, 1319-1325 (2003).

24. Hamm, H., Fabel, H. \& Bartsch, W. The surfactant system of the adult lung: physiology and clinical perspectives. Clin Investig 70, 637-657 (1992).

25. Mason, R.J. \& Williams, M.C. Type II alveolar cell. Defender of the alveolus. Am Rev Respir Dis 115, 81-91 (1977).

26. Lee, J.W., Chou, C.L. \& Knepper, M.A. Deep Sequencing in Microdissected Renal Tubules Identifies Nephron Segment-Specific Transcriptomes. J Am Soc Nephrol 26, 2669-2677 (2015).

27. Santer, R. \& Calado, J. Familial renal glucosuria and SGLT2: from a mendelian trait to a therapeutic target. Clin J Am Soc Nephrol 5, 133-141 (2010).

28. Yu, L. et al. Abnormal expression and dysfunction of novel SGLT2 mutations identified in familial renal glucosuria patients. Hum Genet 129, 335-344 (2011).

29. Chu, P.L. et al. Renal Collectrin Protects against Salt-Sensitive Hypertension and Is Downregulated by Angiotensin II. J Am Soc Nephrol 28, 1826-1837 (2017).

30. Sigdel, T.K. et al. Immune response profiling identifies autoantibodies specific to Moyamoya patients. Orphanet journal of rare diseases 8, 45 (2013).

31. Ball, H.J., Yuasa, H.J., Austin, C.J., Weiser, S. \& Hunt, N.H. Indoleamine 2,3dioxygenase-2; a new enzyme in the kynurenine pathway. The international journal of biochemistry \& cell biology 41, 467-471 (2009).

32. Lepiller, Q. et al. Antiviral and Immunoregulatory Effects of Indoleamine-2,3Dioxygenase in Hepatitis C Virus Infection. J Innate Immun 7, 530-544 (2015).

33. Delmas, B. et al. Aminopeptidase $\mathrm{N}$ is a major receptor for the entero-pathogenic coronavirus TGEV. Nature 357, 417-420 (1992).

34. Wentworth, D.E. \& Holmes, K.V. Molecular determinants of species specificity in the coronavirus receptor aminopeptidase N (CD13): influence of N-linked glycosylation. J Virol 75, 9741-9752 (2001).

35. Guo, Y.R. et al. The origin, transmission and clinical therapies on coronavirus disease 2019 (COVID-19) outbreak - an update on the status. Mil Med Res 7, 11 (2020).

36. Heinrich, P.C., Castell, J.V. \& Andus, T. Interleukin- 6 and the acute phase response. The Biochemical journal 265, 621-636 (1990).

37. Han, X. et al. Mapping the Mouse Cell Atlas by Microwell-Seq. Cell 172, 1091-1107 e1017 (2018).

38. Ong, J., Young, B.E. \& Ong, S. COVID-19 in gastroenterology: a clinical perspective. Gut, Epub ahead of print (2020).

39. Zheng, M. et al. Functional exhaustion of antiviral lymphocytes in COVID-19 patients. Cell Mol Immunol, Epub ahead of print (2020).

40. Wilson, J.G. et al. Mesenchymal stem (stromal) cells for treatment of ARDS: a phase 1 clinical trial. Lancet Respir Med 3, 24-32 (2015).

41. Villiger, P.M. et al. Tocilizumab for induction and maintenance of remission in giant cell arteritis: a phase 2, randomised, double-blind, placebo-controlled trial. Lancet 387, 1921-1927 (2016). 
42. Mosteiro, L., Pantoja, C., de Martino, A. \& Serrano, M. Senescence promotes in vivo reprogramming through p16(INK)(4a) and IL-6. Aging Cell 17 (2018).

43. Wan, S. et al. Characteristics of lymphocyte subsets and cytokines in peripheral blood of 123 hospitalized patients with 2019 novel coronavirus pneumonia (NCP). medRxiv, 2020.2002.2010.20021832 (2020).

44. Shang, J. et al. Structural basis of receptor recognition by SARS-CoV-2. Nature (2020).

45. Bakken, T.E. et al. Single-nucleus and single-cell transcriptomes compared in matched cortical cell types. PLoS One 13, e0209648 (2018).

46. Dobin, A. et al. STAR: ultrafast universal RNA-seq aligner. Bioinformatics 29, 15-21 (2013).

47. Tarasov, A., Vilella, A.J., Cuppen, E., Nijman, I.J. \& Prins, P. Sambamba: fast processing of NGS alignment formats. Bioinformatics 31, 2032-2034 (2015).

48. Wolf, F.A., Angerer, P. \& Theis, F.J. SCANPY: large-scale single-cell gene expression data analysis. Genome Biol 19, 15 (2018).

49. Stuart, T. et al. Comprehensive Integration of Single-Cell Data. Cell 177, 18881902.e1821 (2019).

50. Feng, J., Liu, T., Qin, B., Zhang, Y. \& Liu, X.S. Identifying ChIP-seq enrichment using MACS. Nat Protoc 7, 1728-1740 (2012).

51. Shen, W., Le, S., Li, Y. \& Hu, F. SeqKit: A Cross-Platform and Ultrafast Toolkit for FASTA/Q File Manipulation. PLoS One 11, e0163962 (2016).

52. Stewart, B.J. et al. Spatiotemporal immune zonation of the human kidney. Science 365, 1461-1466 (2019).

53. Madissoon, E. et al. scRNA-seq assessment of the human lung, spleen, and esophagus tissue stability after cold preservation. Genome Biol 21, 1 (2019).

54. Aizarani, N. et al. A human liver cell atlas reveals heterogeneity and epithelial progenitors. Nature 572, 199-204 (2019). 
bioRxiv preprint doi: https://doi.org/10.1101/2020.04.10.022103; this version posted April 24, 2020. The copyright holder for this preprint

(which was not certified by peer review) is the author/funder, who has granted bioRxiv a license to display the preprint in perpetuity. It is made available under aCC-BY-NC-ND 4.0 International license.

a

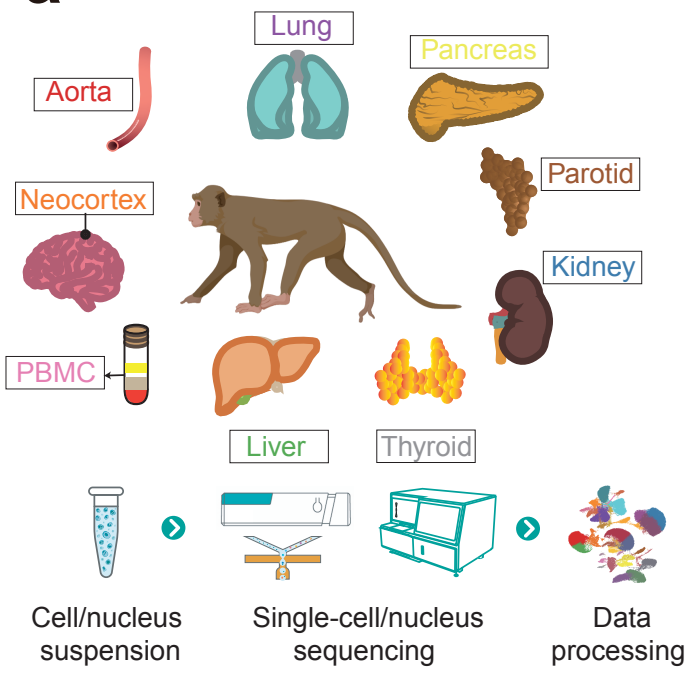

b

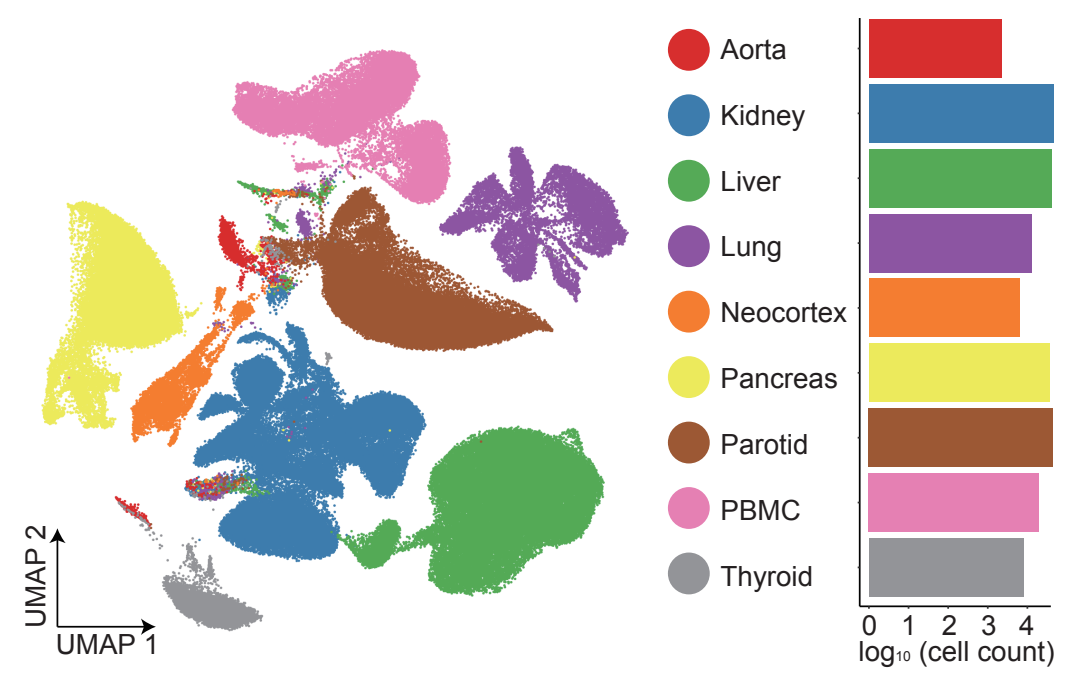

C

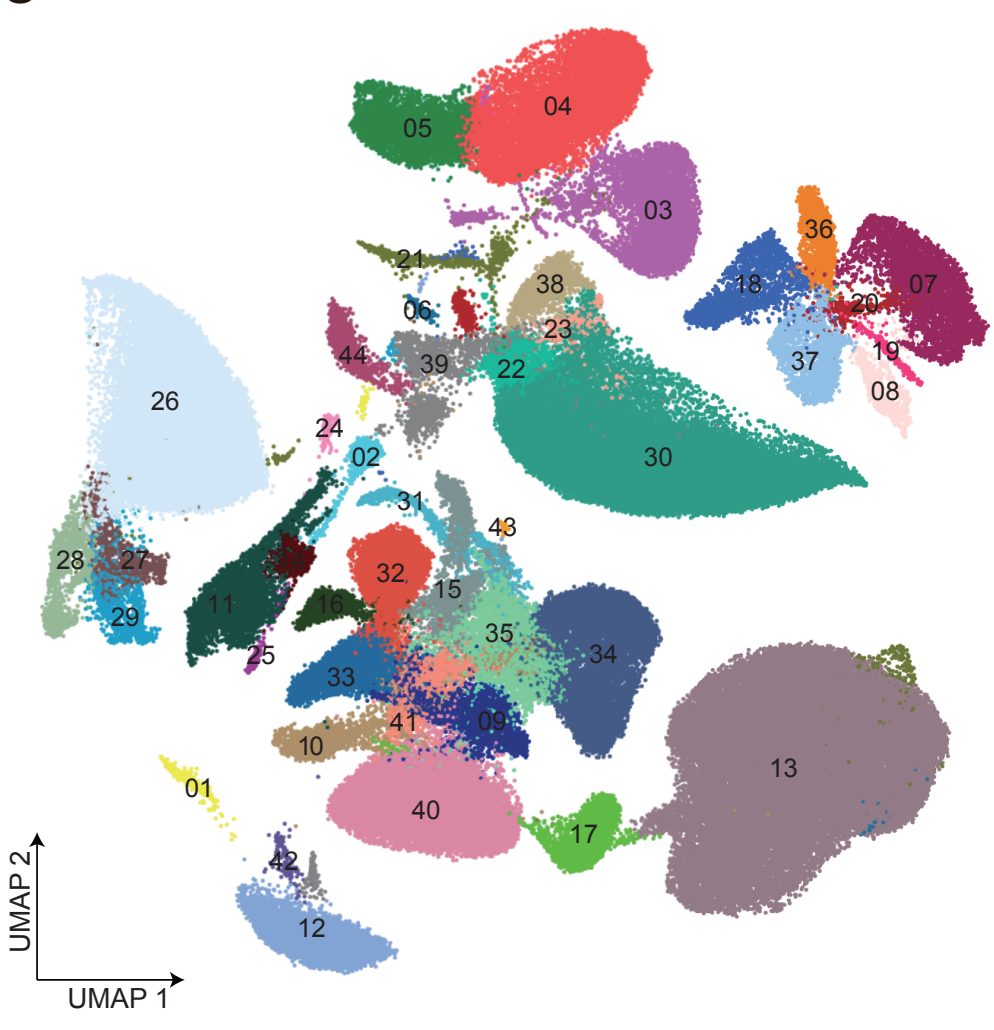

01 Adipocyte

03 B cell

$04-\mathrm{CD}^{+}{ }^{+} \mathrm{T}$ cell

06 Cholangiocyte

07 - Ciliated cell

08 Club cell

- Connecting/Distal convoluted tubule cell

11 - Excitatory neuron

12 - Follicular cell

3. Hepatocyte

4 - Inhibitory neuron

- Intercalated cell 2

17 Liver-endothelial cell

18 - Lung-macrophage

20 - Lung-smooth muscle cell

21 - Macrophage

22 - Mucous acinar cell

23 - Myoepithelial cell

24 - Oligodendrocyte

25 - Oligodendrocyte precursor cell

26 Pancreas-acinar cell

27 - Pancreas-duct cell

28 - Pancreas-islet cell

29 - Pancreas-stromal cell

30 - Parotid-acinar cell

31 - Podocyte

32 - Principal cell 1

33 - Principal cell 2

34 - Proximal tubule cell 1

35 Proximal tubule cell 2

36 - Pulmonary alveolar type 1 cell

37 Pulmonary alveolar type 2 cell

38 Striated duct cell

39 Stromal cell

40 - Thick ascending limb cell

41 Thin limb cell

42

43 muscle cell

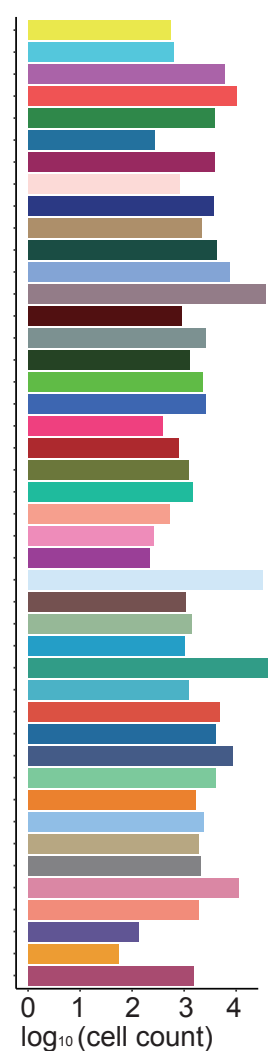


a

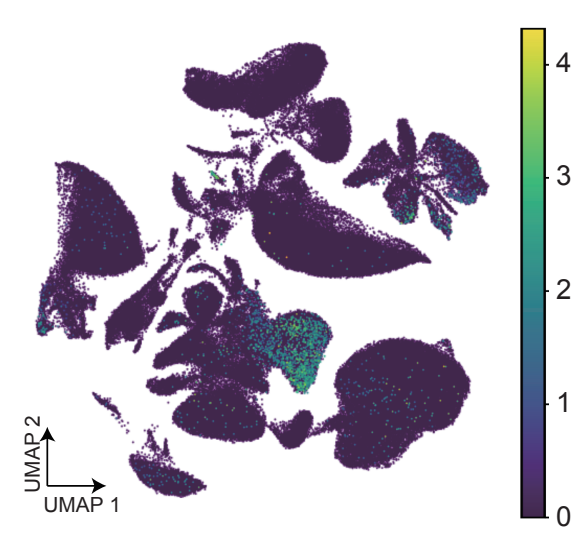

b

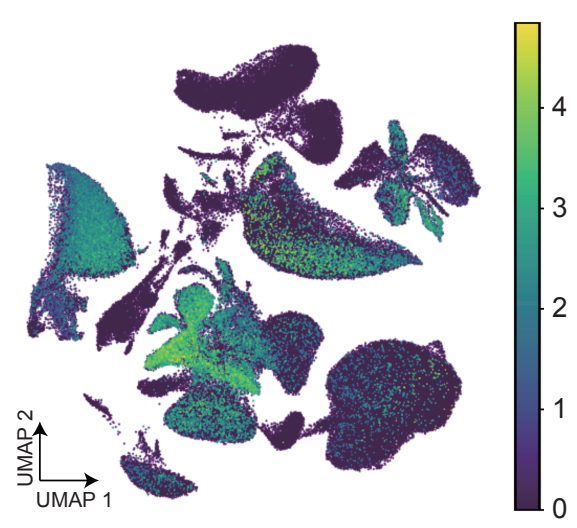

C

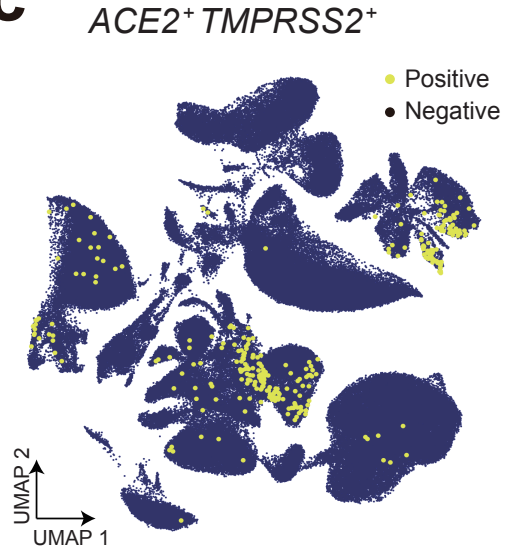

d

ACE2 TMPRSS2

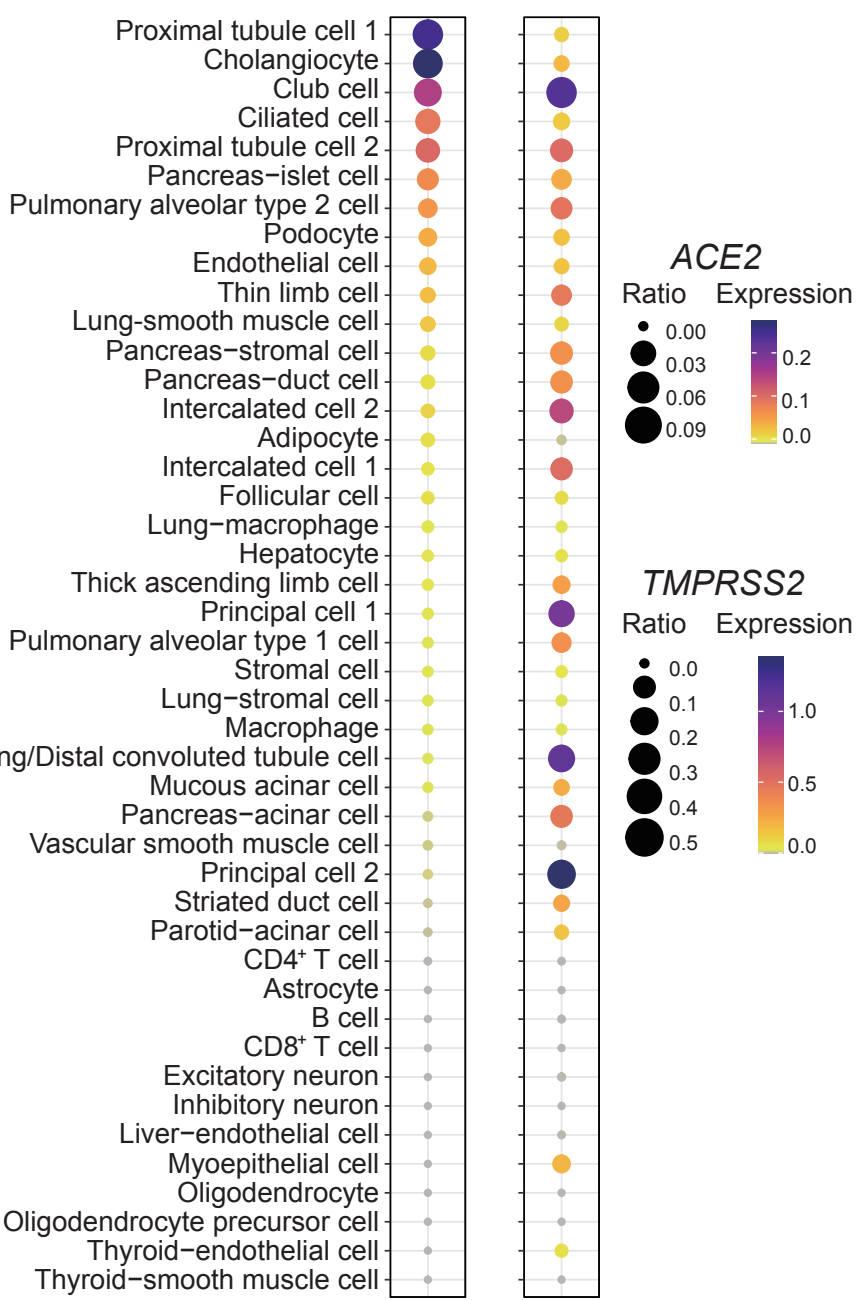

Proportion of $A C E 2^{+}{ }^{T M P R S S 2}{ }^{+}$cells in each cell type

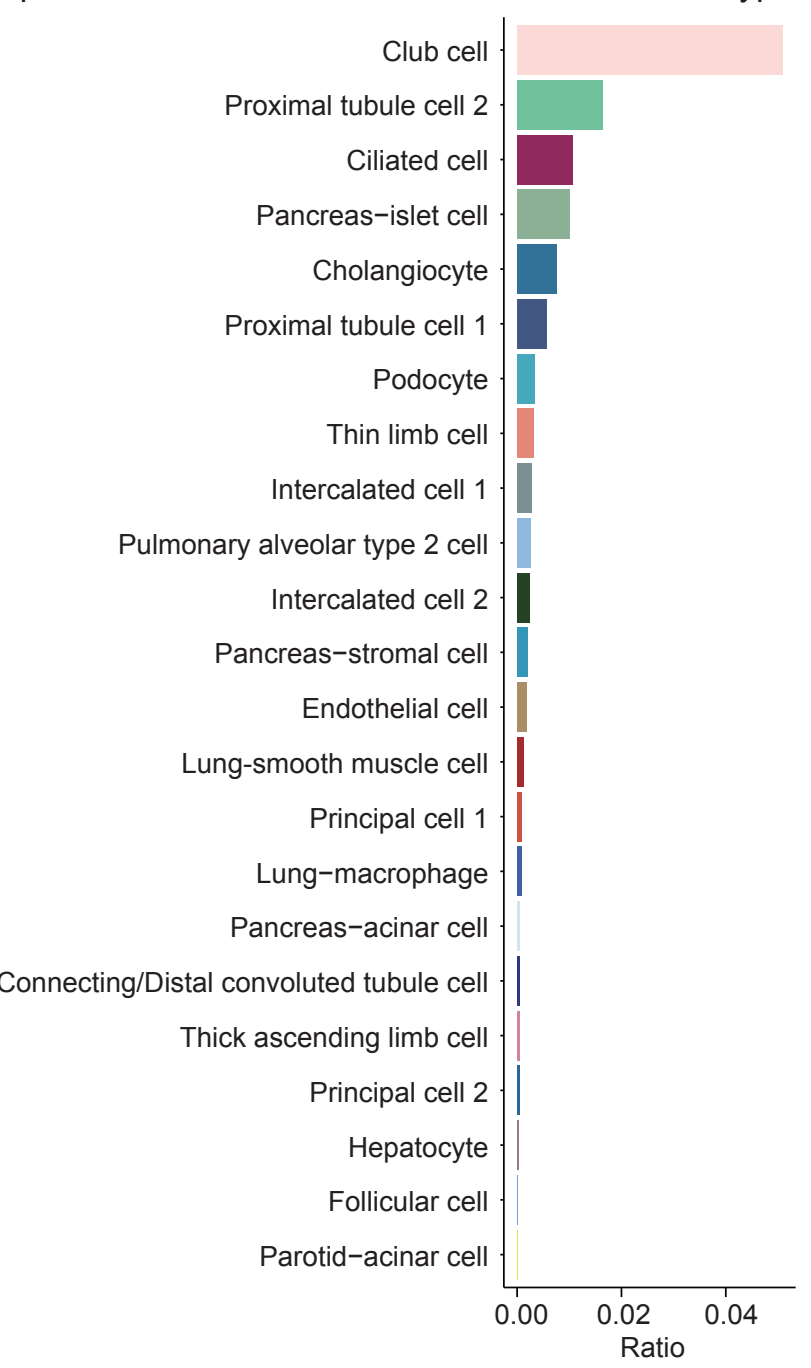


bioRxiv preprint doi: https://doi.org/10.1101/2020.04.10.022103; this version posted April 24, 2020. The copyright holder for this preprint (which was not certified by peer review) is the author/funder, who has granted bioRxiv a license to display the preprint in perpetuity. It is made available under aCC-BY-NC-ND 4.0 International license.

a
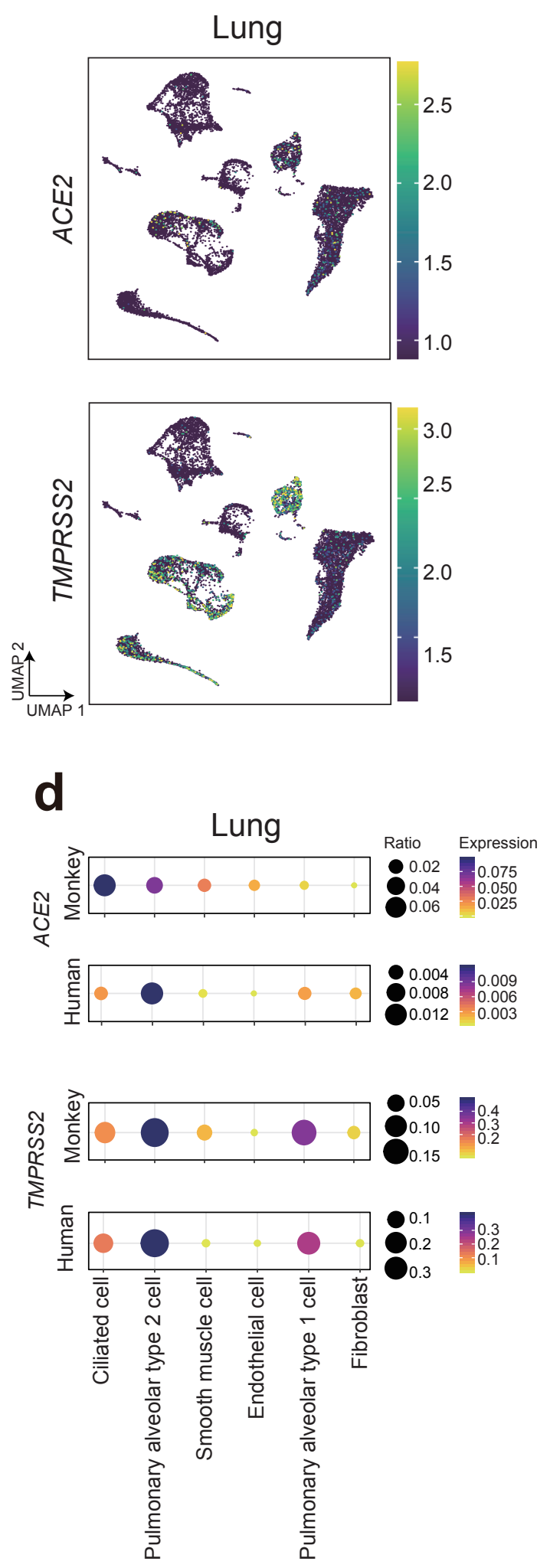

b
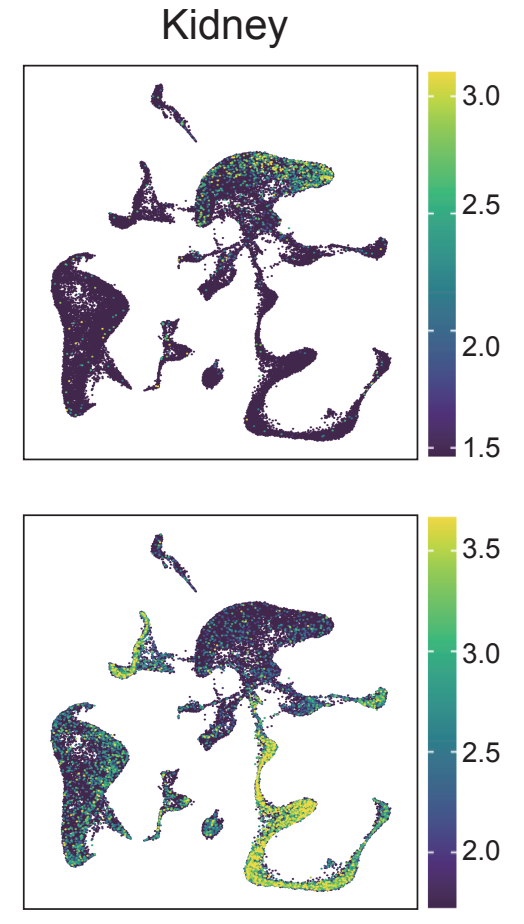

e
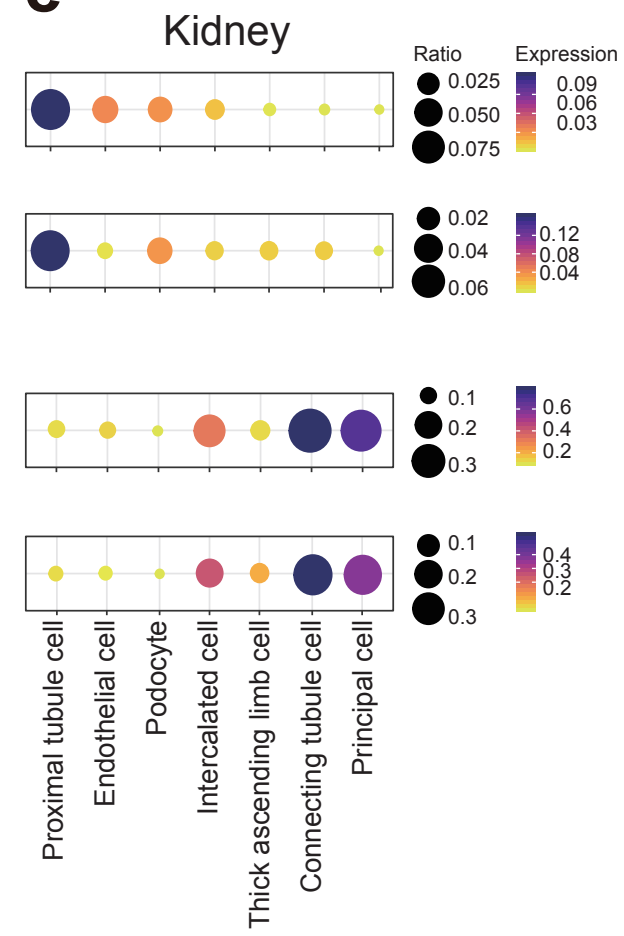

C

Liver
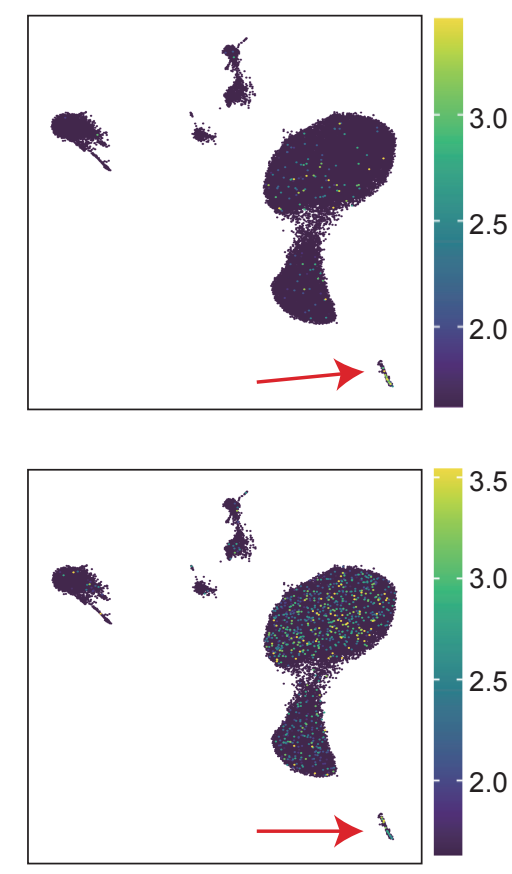

f
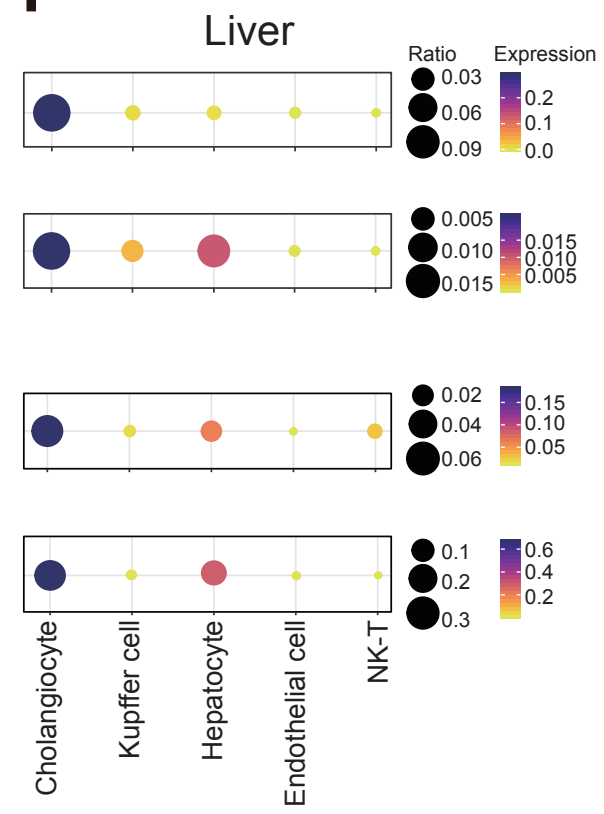
a

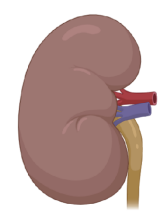

Tn5 transposed single-nuclei
8

C

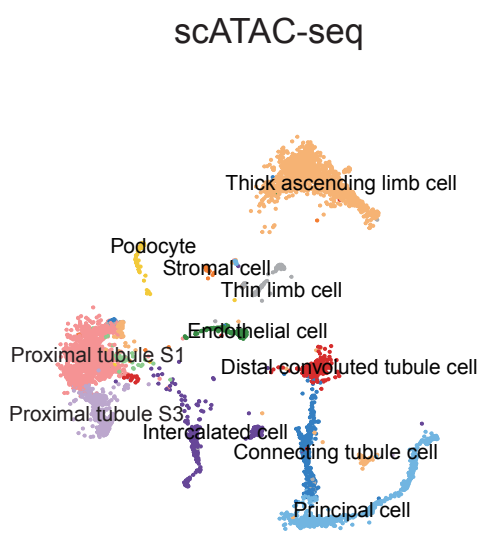

d

\section{Aggregated scATAC-seq}

Connecting tubule cell

Distal convoluted tubule cell

\section{Endothelial cell}

Intercalated cell

Stromal cell

Podocyte

Principal cell

Thick ascending limb cell

Thin limb cell

Proximal tubule $\mathrm{S} 1$

Proximal tubule $\mathrm{S} 3$

f

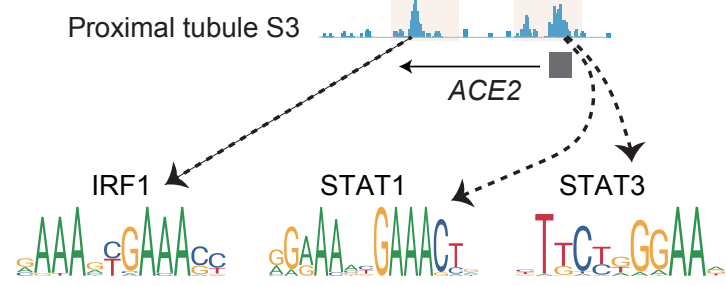

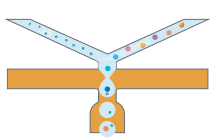

Single-nucleus barcoding $\boldsymbol{\gamma}$

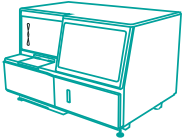

Sequencing

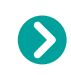

Data processing e

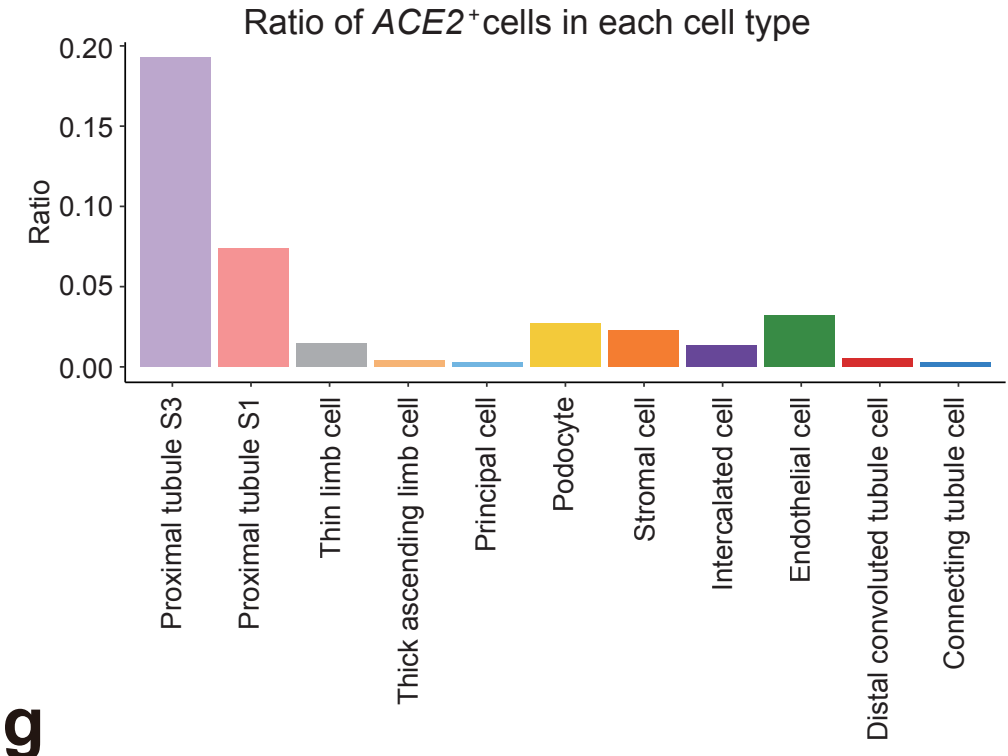

IL6R

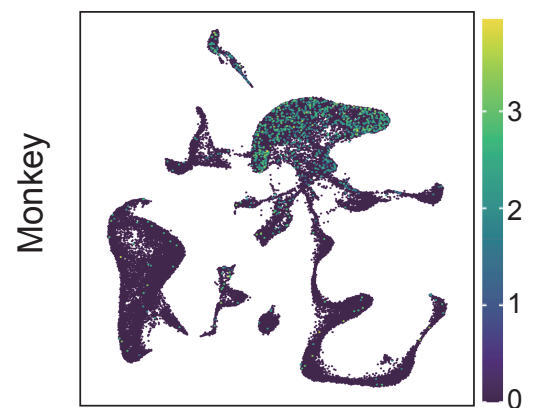

$A C E 2^{+} I^{2} 6 R^{+}$

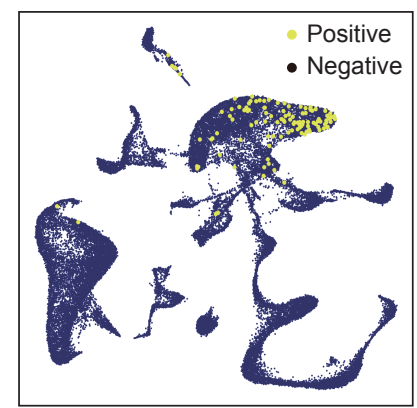

h
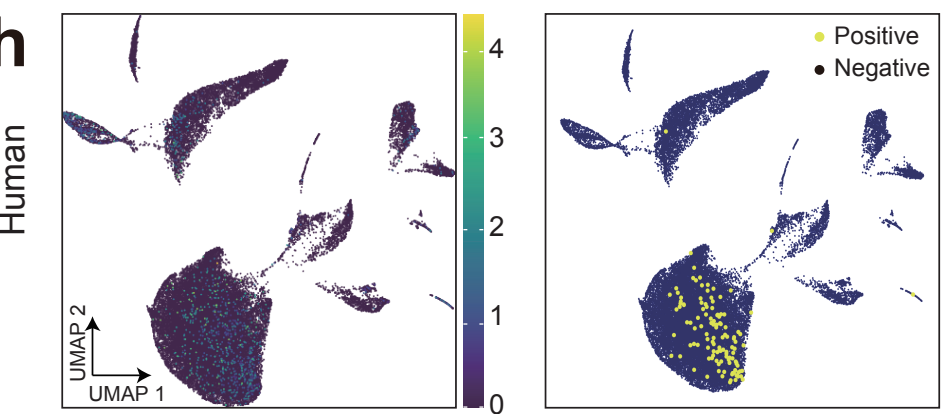


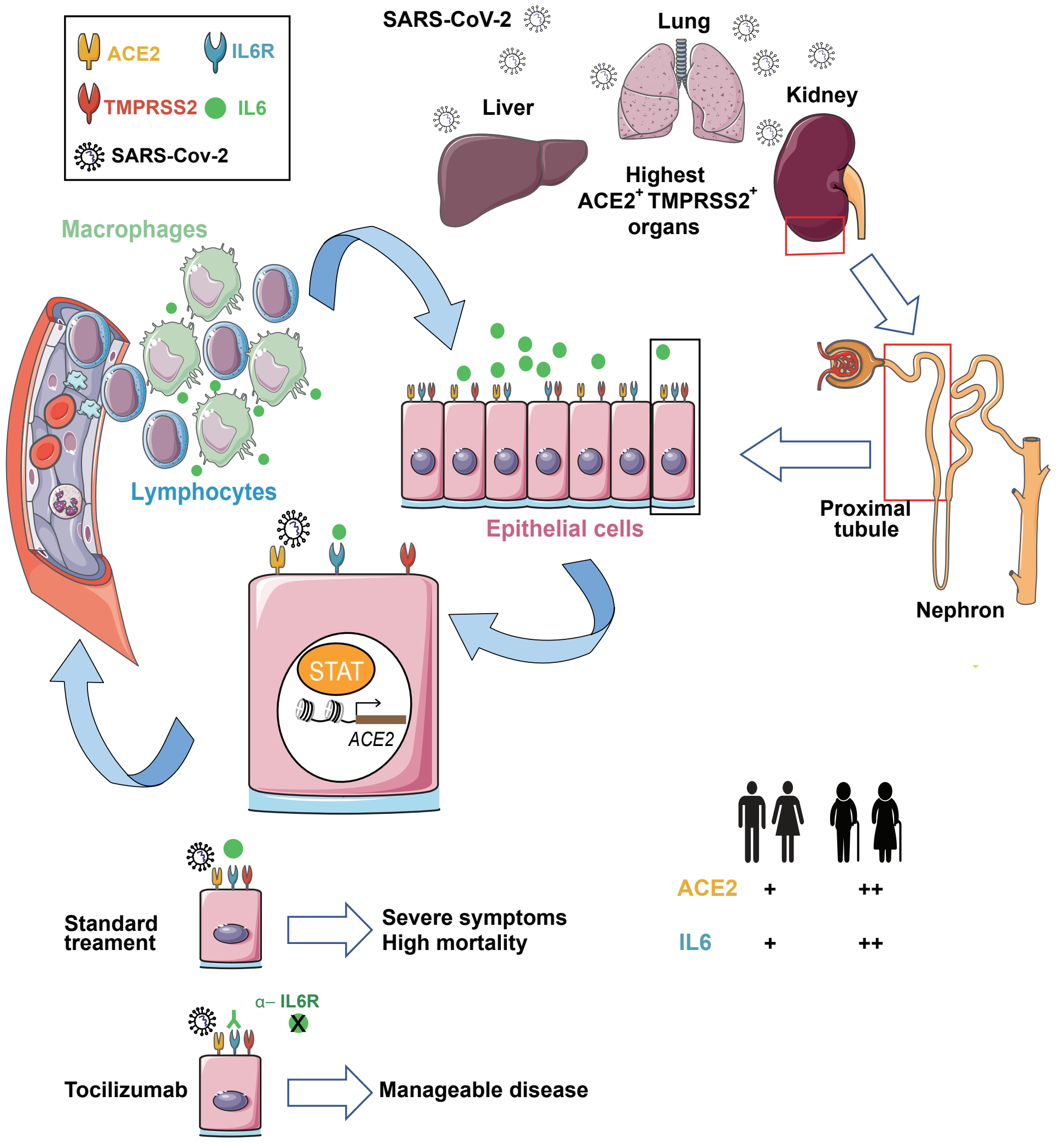


bioRxiv preprint doi: https://doi.org/10.1101/2020.04.10.022103; this version posted April 24, 2020. The copyright holder for this preprint

(which was not certified by peer review) is the author/funder, who has granted bioRxiv a license to display the preprint in perpetuity. It is made available under aCC-BY-NC-ND 4.0 International license.

a

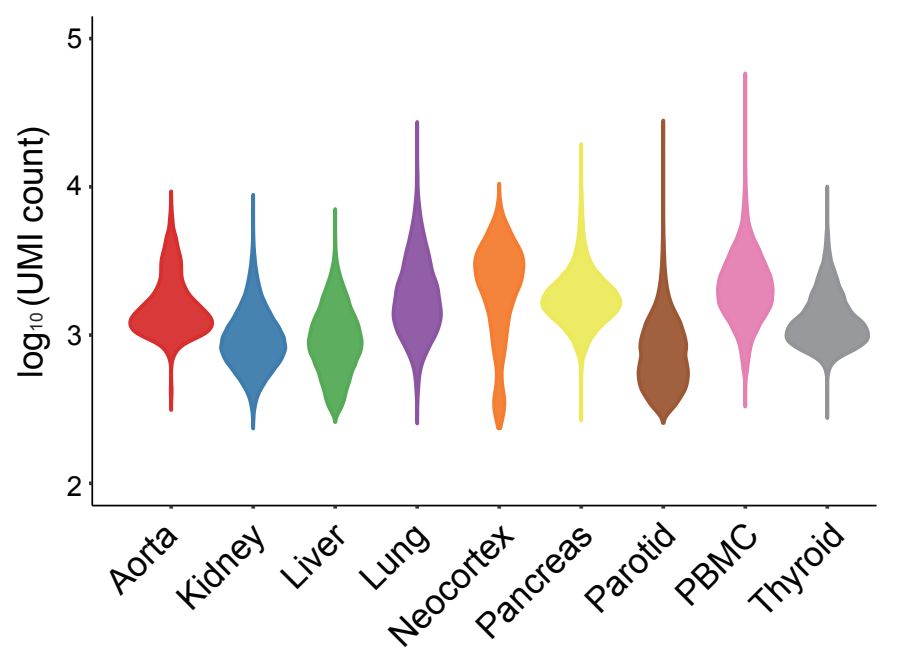

b

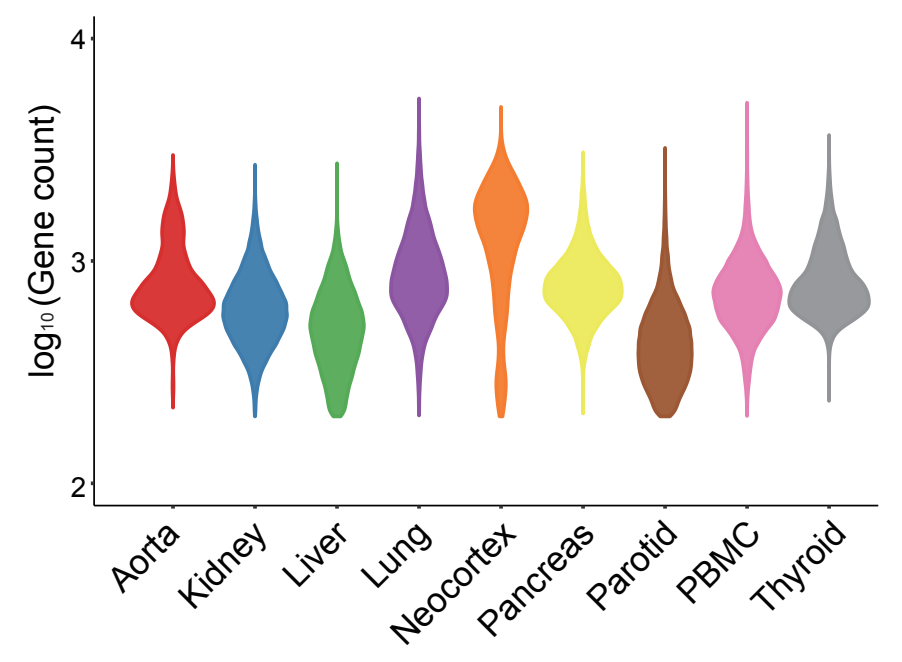

C

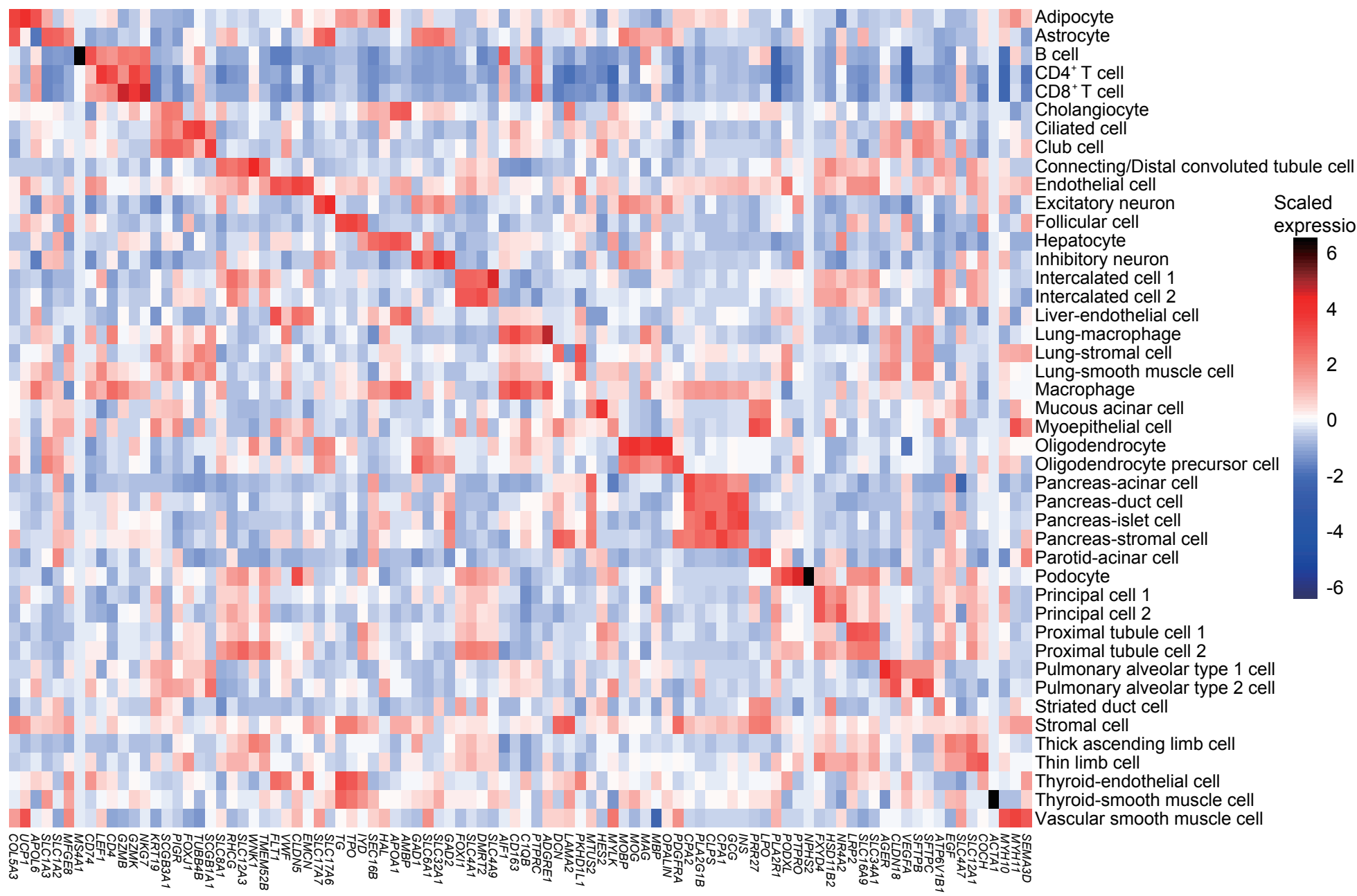


bioRxiv preprint doi: https://doi.org/10.1101/2020.04.10.022103; this version posted April 24, 2020. The copyright holder for this preprint

(which was not certified by peer review) is the author/funder, who has granted bioRxiv a license to display the preprint in perpetuity. It is made available under aCC-BY-NC-ND 4.0 International license.

a

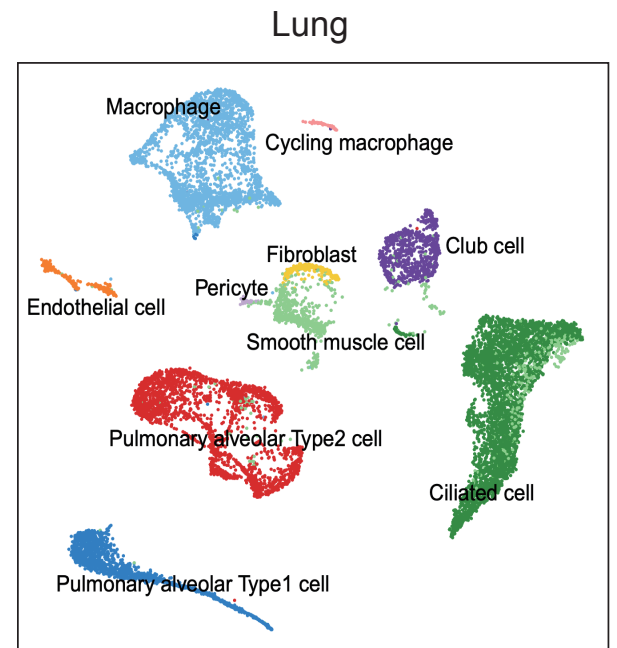

d

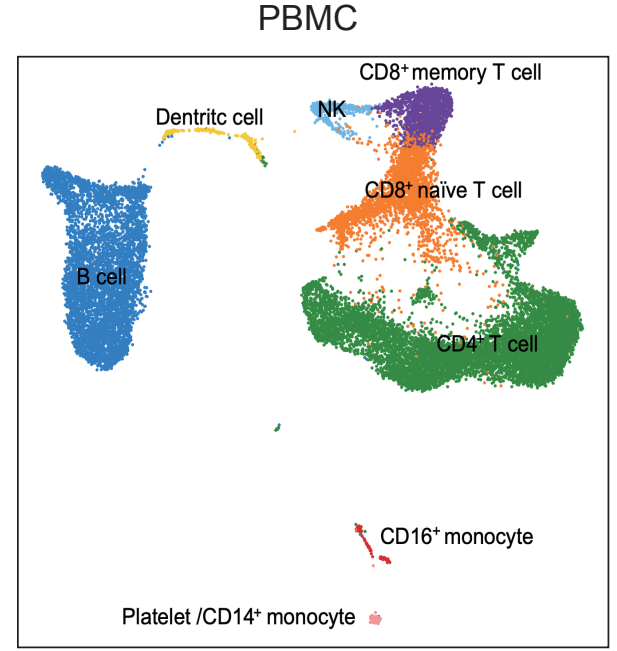

g

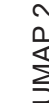

b

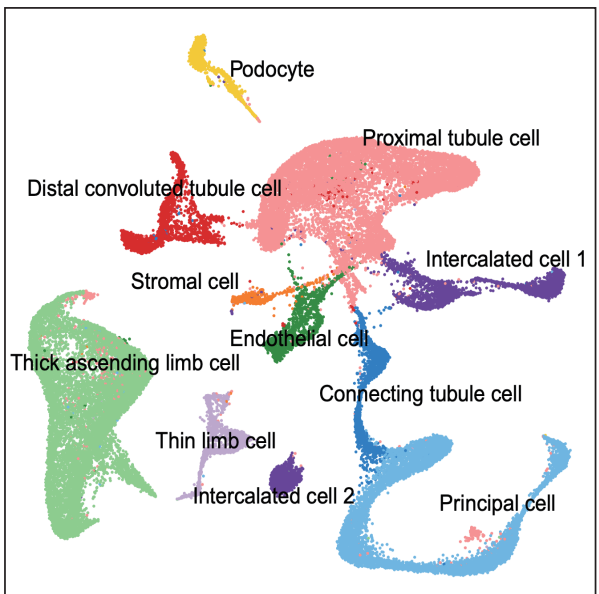

$\mathbf{e}$

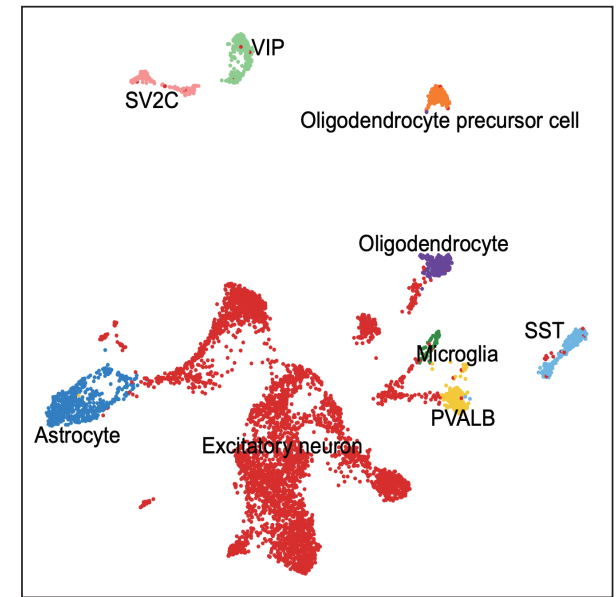

h

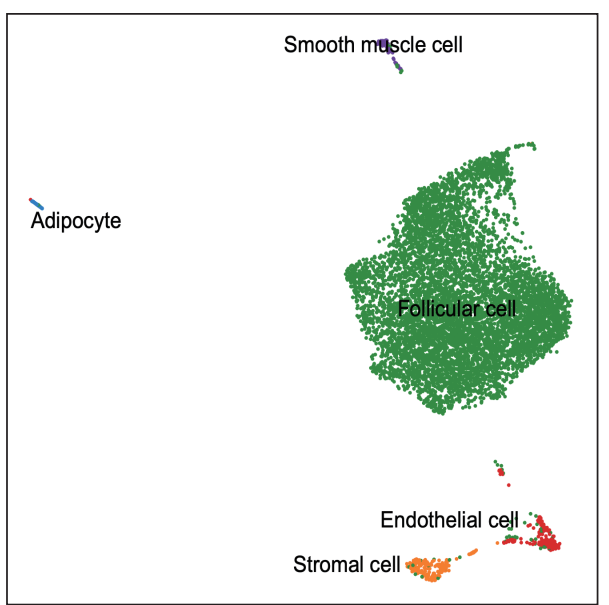

C

Liver

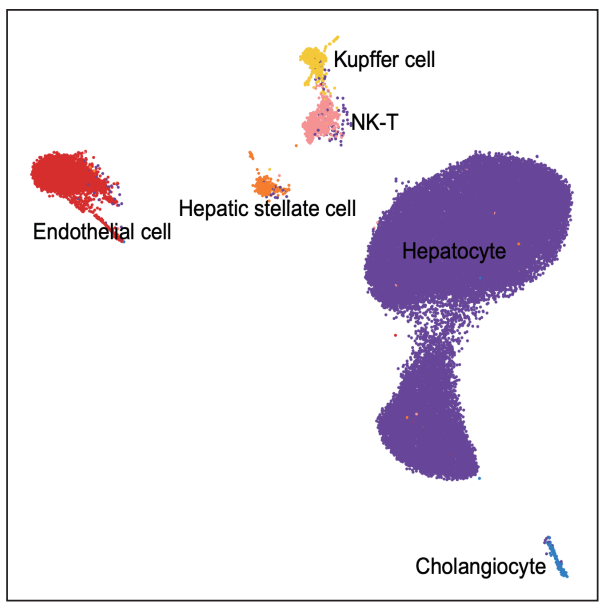

f

Parotid

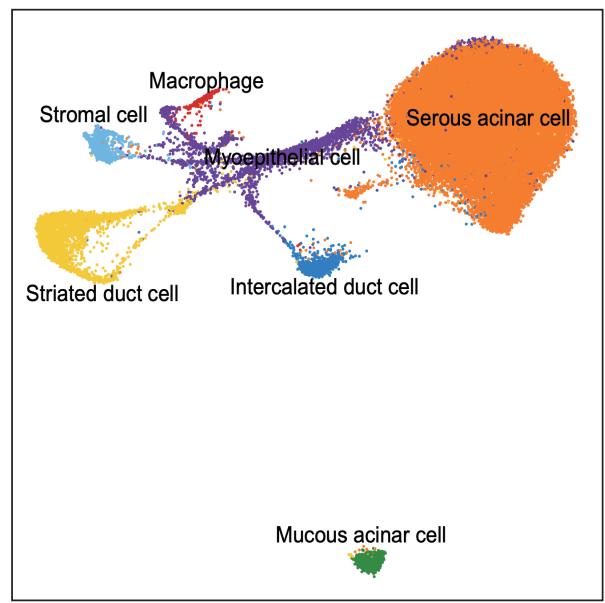

i

Pancreas

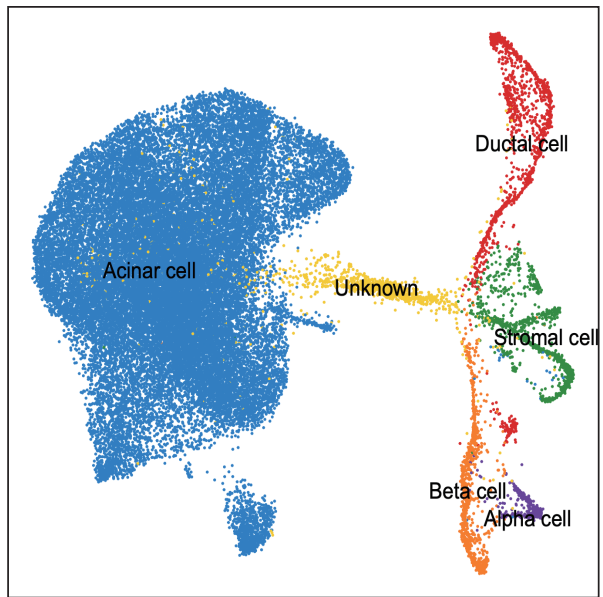


bioRxiv preprint doi: https://doi.org/10.1101/2020.04.10.022103; this version posted April 24, 2020. The copyright holder for this preprint (which was not certified by peer review) is the author/funder, who has granted bioRxiv a license to display the preprint in perpetuity. It is

a

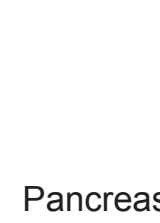

Pans
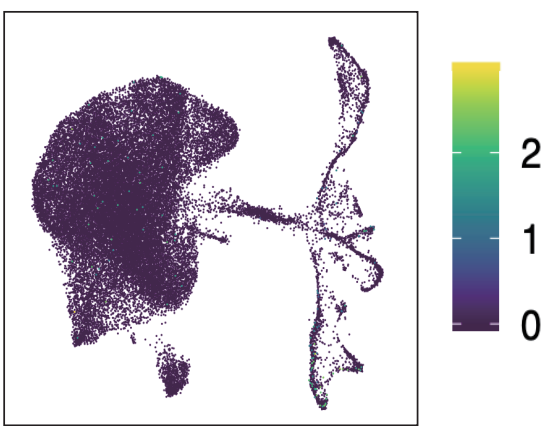

Parotid

PBMC
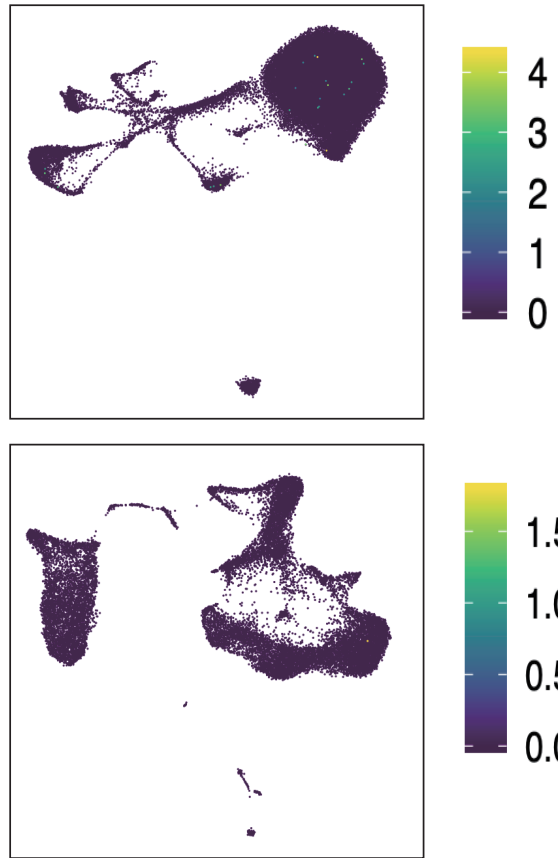

Thyroid
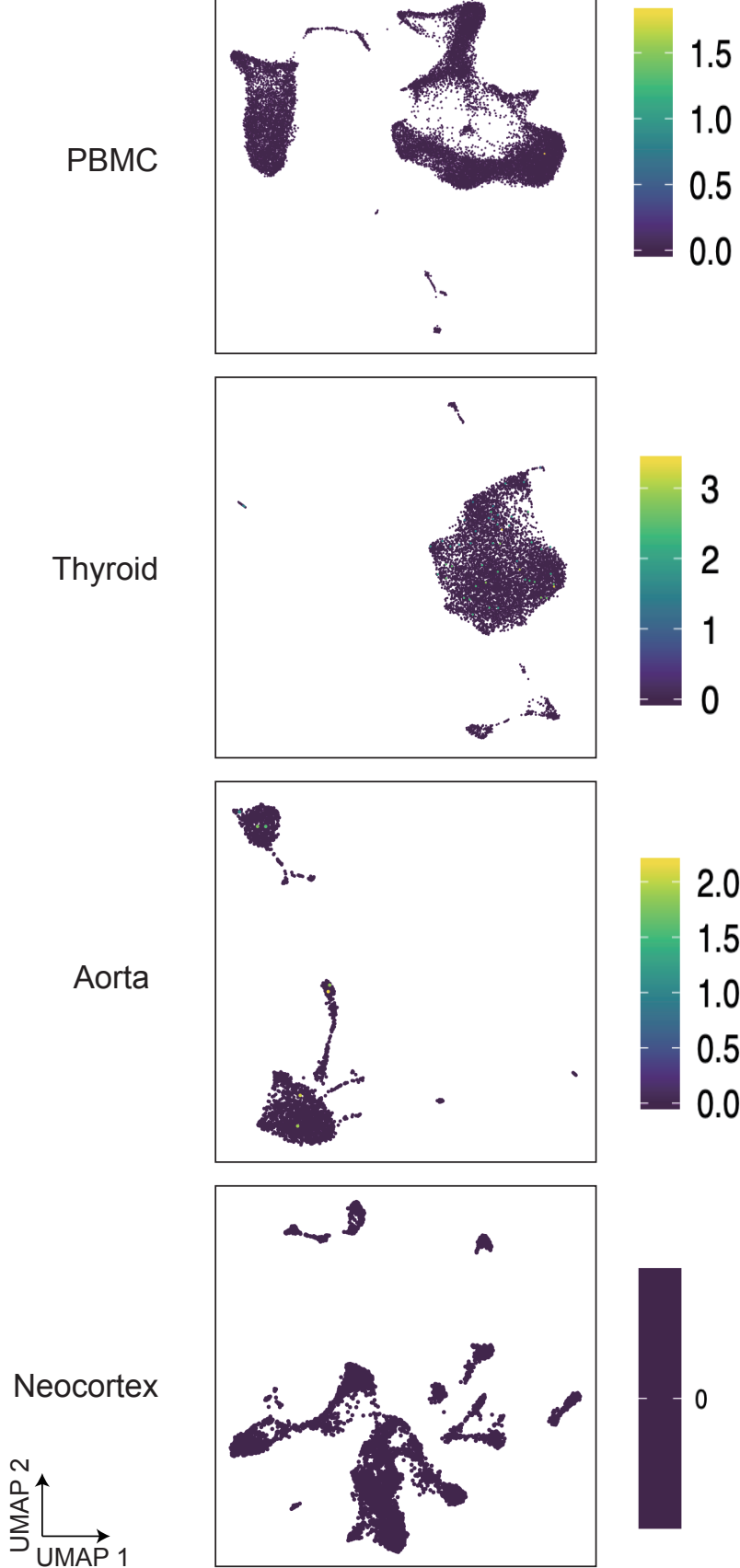
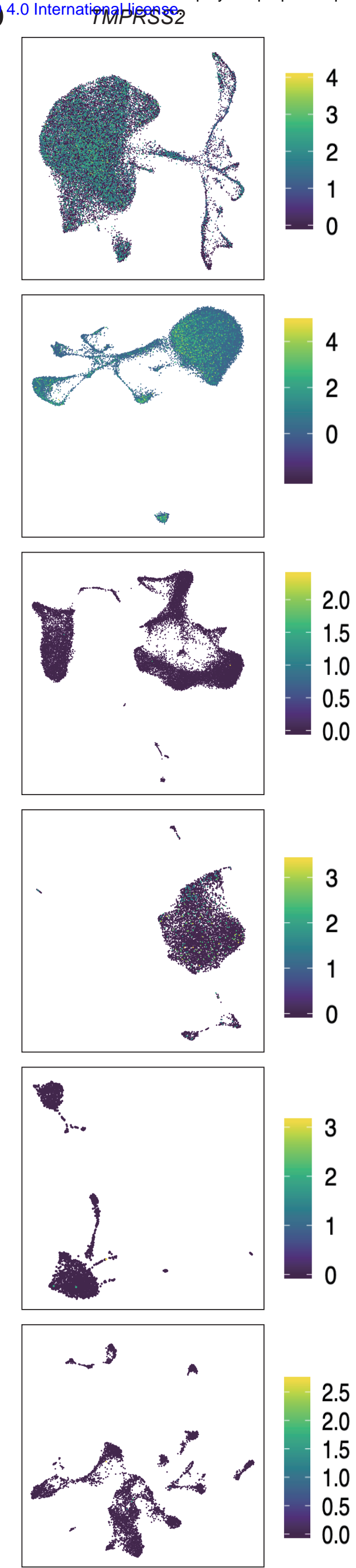
bioRxiv preprint doi: https://doi.org/10.1101/2020.04.10.022103; this version posted April 24, 2020. The copyright holder for this preprint (which was not certified by peer review) is the author/funder, who has granted bioRxiv a license to display the preprint in perpetuity. It is made available under aCC-BY-NC-ND 4.0 International license.

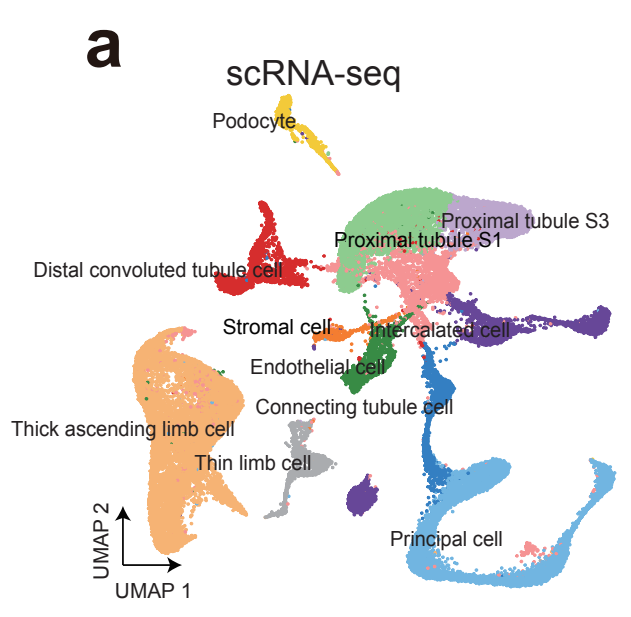

C

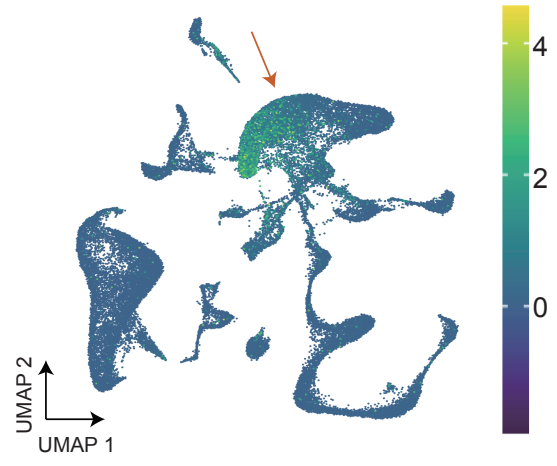

b

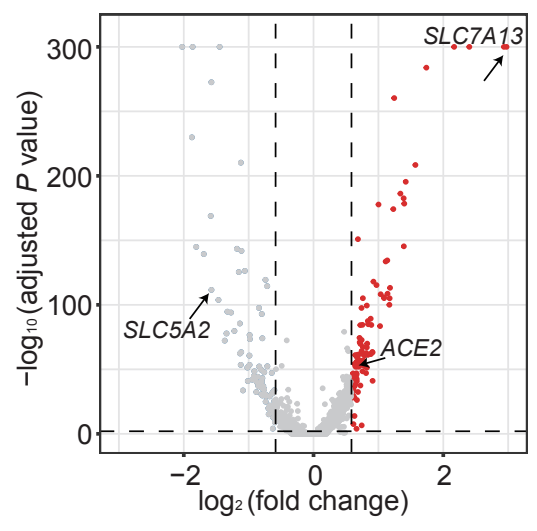

SLC7A13

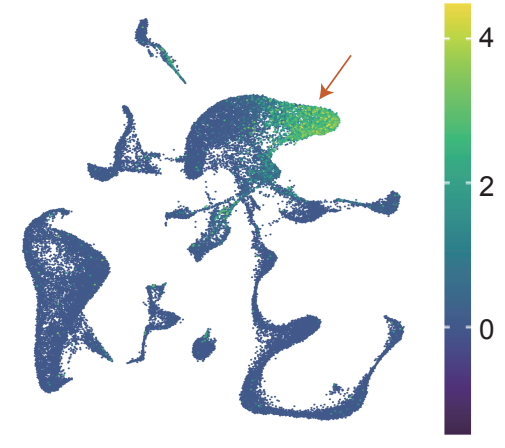

d

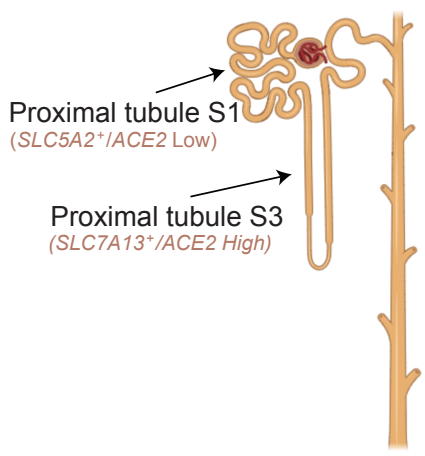


bioRxiv preprint doi: https://doi.org/10.1101/2020.04.10.022103; this version posted April 24, 2020. The copyright holder for this preprint

(which was not certified by peer review) is the author/funder, who has granted bioRxiv a license to display the preprint in perpetuity. It is made available under aCC-BY-NC-ND 4.0 International license.

a

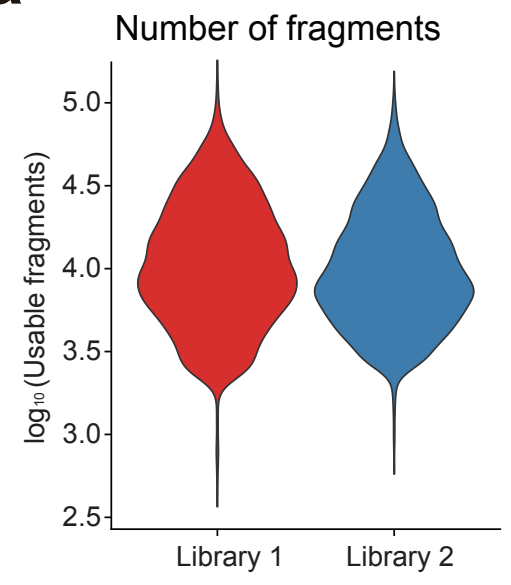

b

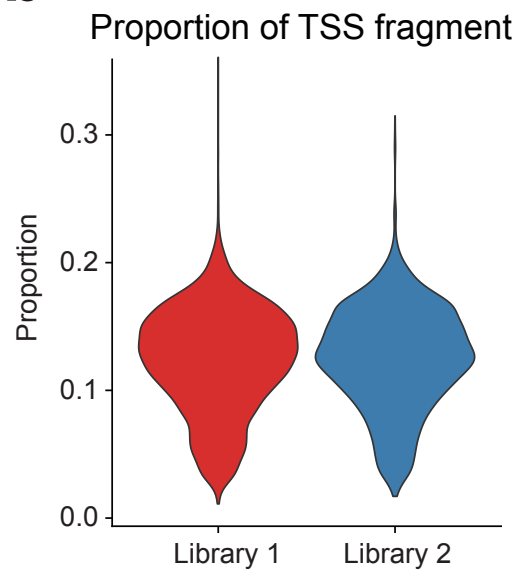

d

Human ACE2

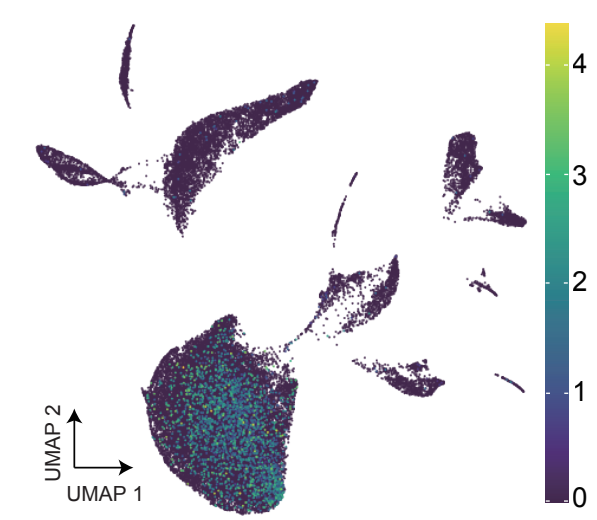

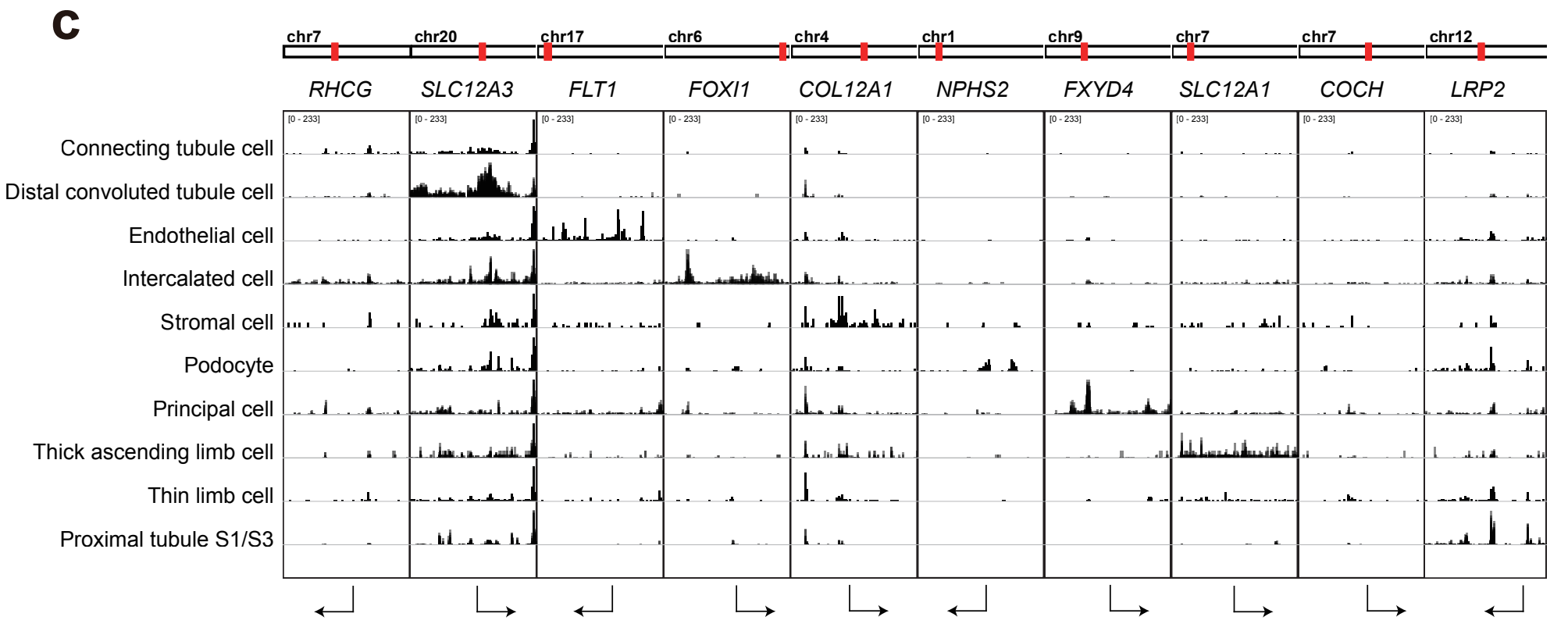

